ARTICLE

\title{
Nanoparticle-assembled bioadhesive coacervate coating with prolonged gastrointestinal retention for inflammatory bowel disease therapy
}

\author{
Pengchao Zhao ${ }^{1,7}$, Xianfeng Xia (10) ${ }^{2,3,7}$, Xiayi Xu ${ }^{1,7}$, Kevin Kai Chung Leung ${ }^{4}$, Aliza Rai ${ }^{4}$, Yingrui Deng ${ }^{1}$, \\ Boguang Yang${ }^{1}$, Huasheng Lai ${ }^{4}$, Xin Peng ${ }^{1}$, Peng Shi ${ }^{5,6}$, Honglu Zhang ${ }^{5,6}$, Philip Wai Yan Chiu (1) ${ }^{1,3,4 凶} \&$ \\ Liming Bian (iD) 5,6凶
}

A key challenge for the effective treatment of gastrointestinal diseases including inflammatory bowel disease is to develop an orally administered drug delivery system capable of prolonged retention in the gastrointestinal tract. Herein we report a bioadhesive liquid coacervate based on hydrogen bonding-driven nanoparticle assembly. Free from electrostatic interactions, our fluid nanoparticle-assembled coacervate demonstrates significant $\mathrm{pH}$ - and salt-independent structural stability and forms a physically adhesive coating on a large surface area of intestinal tract with an extended residence time of more than 2 days to mediate the sustained release of preloaded water-soluble small molecule drugs in vivo. The orally administered drug-laden nanoparticle-assembled coacervate significantly mitigates the symptoms of inflammatory bowel disease, restores the diversity of gut microbiota, reduces systemic drug exposure, and improves the therapeutic efficacy in a rat acute colitis model compared with the oral administration of the same amount of drug in solution form. We suggest that the nanoparticle-assembled coacervate provides a promising drug delivery platform for management and treatment of numerous gastrointestinal diseases where controlled drug release with extended residence time is desired.

\footnotetext{
${ }^{1}$ Department of Biomedical Engineering, The Chinese University of Hong Kong, Hong Kong 999077, China. ${ }^{2}$ Department of Endoscopy, State Key Laboratory of Oncology in South China, Collaborative Innovation Center for Cancer Medicine, Sun Yat-sen University Cancer Center, Guangzhou 510000, China. ${ }^{3}$ Chow Yuk Ho Technology Centre for Innovative Medicine, The Chinese University of Hong Kong, Hong Kong 999077, China. ${ }^{4}$ Department of Surgery, Institute of Digestive Disease, State Key Laboratory of Digestive Disease, The Chinese University of Hong Kong, Hong Kong 999077, China. ${ }^{5}$ School of Biomedical Sciences and Engineering, Guangzhou International Campus, South China University of Technology, Guangzhou 511442, China. ${ }^{6}$ National Engineering Research Center for Tissue Restoration and Reconstruction, South China University of Technology, Guangzhou 510006, China. ${ }^{7}$ These authors contributed equally: Pengchao Zhao, Xianfeng Xia, Xiayi Xu. 凶email: philipchiu@surgery.cuhk.edu.hk; bianlm@scut.edu.cn
} 
nflammatory bowel disease (IBD) including Crohn's disease (CD) and ulcerative colitis (UC) causes chronic relapsing inflammation of the gastrointestinal (GI) tract ${ }^{1}$. IBD significantly increases the risk of developing colorectal cancer and imposes a lifelong healthcare burden on millions of patients worldwide $^{2-4}$. Oral dosage formulations of drugs such as corticosteroids are the most desirable delivery systems available to treat IBD and are vastly superior to enema and subcutaneous and intravenous injection in terms of patient compliance ${ }^{5}$. However, many patients require lifelong administration of drugs to control IBD symptoms; therefore, reducing the severity of side effects attributable to systemic drug exposure resulting from frequent oral dosing via conventional delivery systems, such as entericcoated tablets and capsules, is essential ${ }^{6}$.

Although many forms of oral drug delivery vehicles have been developed, the insufficient residence time within the intestinal tract and the complex design of delivery vehicles limit their widespread clinical applications ${ }^{7-10}$. Endowing oral drug delivery vehicles with wet bioadhesion may prolong intestinal residence time. However, solid adhesives with high adhesion energy on wet tissues ${ }^{11-14}$ may not be suitable as orally administered intestine-coating drug delivery platforms. For example, we recently reported a bioadhesive hydrogel, which can adhere to gastric ulcer sites upon in situ gelation ${ }^{15}$. However, such adhesive hydrogel cannot form the extensive and uniform adhesive coating layer on the convoluted intestinal tract surfaces due to its solid nature after rapid gelation.

Distinct from the solid bioadhesives, conventional complex coacervates are polymer-concentrated and water-immiscible liquids formed by fluid-fluid phase separation of complexed polyanions and polycations and represent an exciting class of drug delivery vehicles $^{16-18}$. However, the complex coacervation process is driven by electrostatic attraction between polyanions and polycations within narrow $\mathrm{pH} / \mathrm{salt}^{\text {ranges }}{ }^{19}$. Considering that the GI tract routinely undergoes substantial changes in motility, fluid content, and acidity (from $\mathrm{pH} 1.5$ in the stomach to $\mathrm{pH} 6.15-7.88$ in intestines) $)^{5,20}$, conventional complex coacervates can be easily disrupted by $\mathrm{pH} /$ salt variations (Fig. $1 \mathrm{a}, \mathrm{b})^{21-23}$. One recent groundbreaking work reported the precise engineering of low molecular weight sucralfate into complex coacervate to increase the residence time in intestines to several hours ${ }^{24}$. Despite these advances, the limited choices of polyelectrolytes species for preparing complex coacervates that can adapt to the harsh in vivo environment, together with the insufficient intestinal residence time, greatly hamper the development of fluid coacervates as the orally administrated drug delivery systems.

Here we report a universal strategy to prepare water-immiscible, bioadhesive, and noncomplex liquid coacervates derived from bidentate hydrogen bonding-driven self-assembly of nanoparticles as an orally administered intestinal-coating formulation (Fig. $1 \mathrm{~b}$ and Supplementary Fig. 1). Driven by the gastrointestinal peristalsis, the nanoparticle-assembled fluid coacervate (named NPA coacervate hereafter) can effectively spread to coat and adhere on the large intestinal surface area with a prolonged residence time of more than 2 days to mediate the sustained release of loaded drugs (Fig. 1c). Using the water-soluble dexamethasone sodium phosphate (Dex-P) as a model small molecule drug, we demonstrated that the oral administration of drugladen NPA coacervate significantly enhanced the IBD therapeutic outcomes, improved the richness and diversity of gut microbiota, and reduced the systemic drug exposure compared with the treatment with Dex-P aqueous solution in a rat model of dextran sulfate sodium (DSS)-induced acute colitis-one of the most widely used models due to its shared clinical and histopathological characteristics with human $\mathrm{IBD}^{25}$ and its validation as a relevant model to treat human diseases ${ }^{26}$.

\section{Results and discussion}

The design of noncomplex NPA coacervates via nanoparticle assembly. Nanoparticles required for fabricating NPA coacervates contain a hydrophobic core and end-functionalized (e.g., catechol) hydrophilic polyethylene glycol (PEG) chains as the shell (Fig. 1d). In this study, MALDI-TOF mass spectrometry was used to confirm the successful synthesis of the hydrophobic core (Supplementary Fig. 2). The molecular weight distribution of the hydrophilic PEG chains was further determined by gel permeation chromatography (GPC, Supplementary Fig. 2). Dynamic light scattering (DLS) analysis confirmed the formation of the asprepared $\mathrm{NP}_{1}, \mathrm{NP}_{2}$, and $\mathrm{NP}_{3}$ nanoparticles with hydrodynamic radii of $\sim 50-150 \mathrm{~nm}$ (Supplementary Fig. 3). The bidentate hydrogen bonding and $\pi-\pi$ interactions derived from the catechol $^{27-29}$ and phenyl ${ }^{30,31}$ end groups of the hydrophilic PEG chains provided the driving force to induce the assembly of $\mathrm{NP}_{2}$ and $\mathrm{NP}_{3}$ nanoparticles and formation of the liquid $\mathrm{NPA}_{2}$ and $\mathrm{NPA}_{3}$ coacervates $\left(\mathrm{G}^{\prime}<\mathrm{G}^{\prime \prime}\right)$, respectively (Fig. $1 \mathrm{~d}$ and Supplementary Fig. 4$)^{32-34}$. In contrast, the control $\mathrm{NP}_{1}$ nanoparticles with no end groups failed to form coacervates via fluid-fluid phase separation, thereby confirming the importance of the physical interactions of end groups to the formation of NPA coacervates (Supplementary Fig. 5).

The $\mathrm{NPA}_{2}$ coacervate was chosen as the model for further investigations because hydrogen-bonding interactions provided by the functionalized catechol groups that drive the coacervation of $\mathrm{NP}_{2}$ nanoparticles can mediate adhesion of the $\mathrm{NPA}_{2}$ coacervate to wet tissue surfaces ${ }^{35-38}$. The coacervation behaviors of $\mathrm{NPA}_{2}$ can be visualized by fluid-fluid phase separation and further examined by transmission electron microscopy (TEM), Fourier-transform infrared spectroscopy (FTIR), and DLS (Fig. 1e). The dark spots marked by red circles in the TEM images are supposed to be the hydrophobic cores of the assembled $\mathrm{NP}_{2}$ nanoparticles, while the catechol-functionalized hydrophilic PEG shell acts as a flexible polymer matrix that connects the hydrophobic cores to form the stable $\mathrm{NPA}_{2}$ coacervate dense phase. It is noted that the free hydroxyl $(-\mathrm{OH})$ groups generally exhibit narrow and intense absorption bands, while -OH groups involved in hydrogen bonding show a broader absorption band in FTIR ${ }^{39,40}$. The FTIR result of freeze-dried $\mathrm{NPA}_{2}$ coacervate showed an obvious broad -OH band, indicating the existence of hydrogen bonding interactions derived from catechol groups (Supplementary Fig. 6). DLS analysis further showed hydrogen bonding-mediated assembly of $\mathrm{NP}_{2}$ nanoparticles to form coacervate micro-droplets as confirmed by the increasing hydrodynamic radius of $\mathrm{NP}_{2}$ nanoparticles after $3.5 \mathrm{~h}$ of dialysis (Supplementary Fig. 7).

Free from electrostatic interactions, our noncomplex $\mathrm{NPA}_{2}$ coacervate is believed to be $\mathrm{pH}$ - and salt-independent. Compared with conventional complex coacervates that depend on $\mathrm{pH}$ values ${ }^{21}$, our $\mathrm{NPA}_{2}$ coacervate remained stable after 2 days and did not become a single-phase solution under a wide range of $\mathrm{pH}$ conditions (Fig. 1f). The frequency-dependent storage $\left(\mathrm{G}^{\prime}\right)$ and loss $\left(\mathrm{G}^{\prime \prime}\right)$ moduli of the liquid $\mathrm{NPA}_{2}$ coacervate as revealed by rheological analysis demonstrates its highly dynamic polymeric structure due to the presence of supramolecular interactions, such as hydrogen bonding derived from the catechol groups (Supplementary Fig. 8) ${ }^{41}$. Furthermore, our noncomplex $\mathrm{NPA}_{2}$ coacervate exhibited a salting-out effect $^{42,43}$, and the shear moduli $\left(G^{\prime}\right.$ and $\left.G^{\prime \prime}\right)$ and viscosity increased with increasing salt concentrations (Fig. 1g and Supplementary Fig. 9). It should be noted that electrostatic interactions between polyanions and polycations weaken with increasing salt concentrations; therefore, a critical salt concentration of $0.8-2.0 \mathrm{M} \mathrm{NaCl}$ generally can lead to the rapid dissociation of complex coacervates ${ }^{19,22}$. However, our $\mathrm{NPA}_{2}$ coacervate remained as a viscous liquid $\left(\mathrm{G}^{\prime}<\mathrm{G}^{\prime \prime}\right)$ in 
a

Harsh environmental conditions in peristaltic GI tract

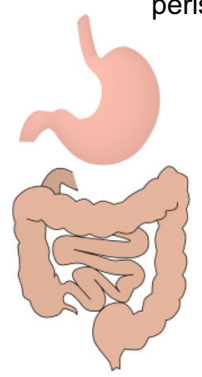

Stomach

pH 1-2.5; Pepsin, lipase and exopeptidase

Small intestine

pH 6.15-7.88; Enzymes primarily from pancreas

Colon

pH 5.26-7.2; Bacterial enzymes
C

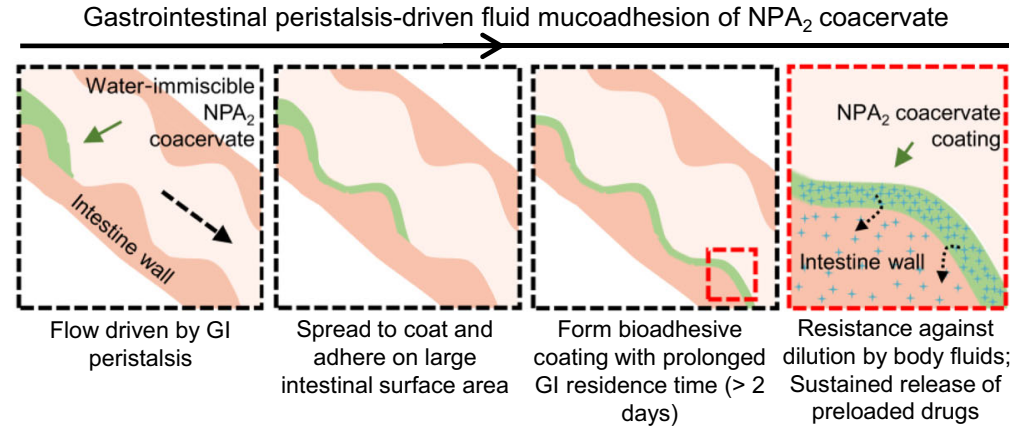

b

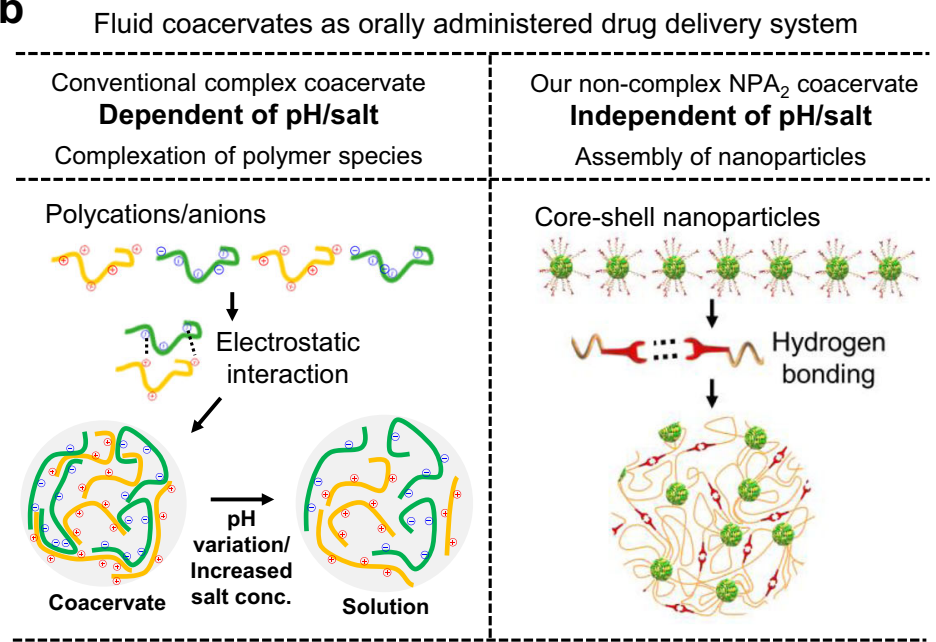

d

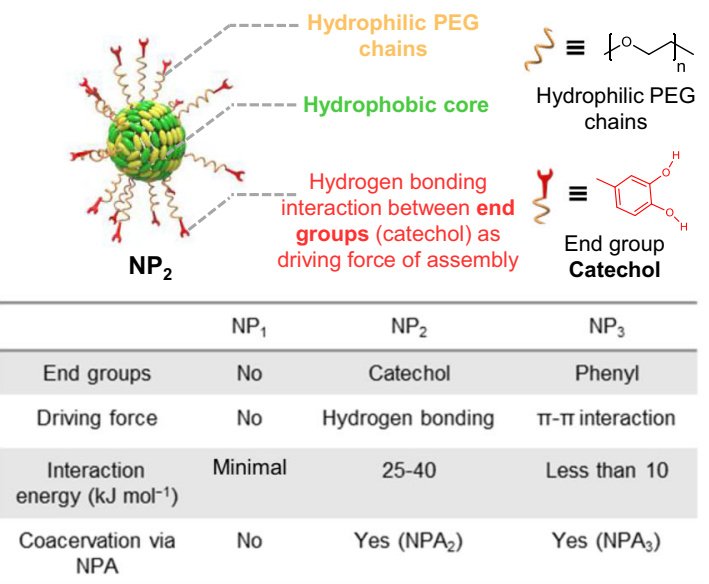

e

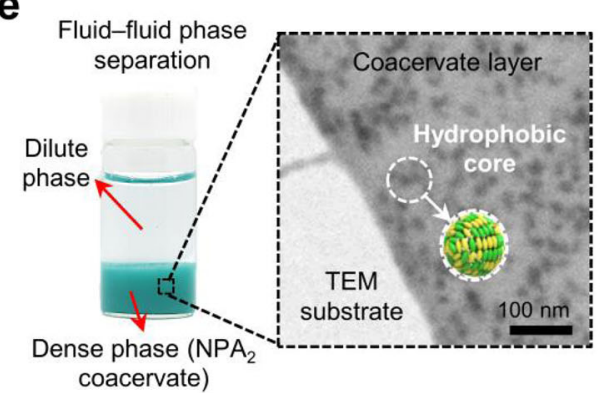

$f$

f $\mathrm{pH}$-independent $\mathrm{NPA}_{2}$ coacervate

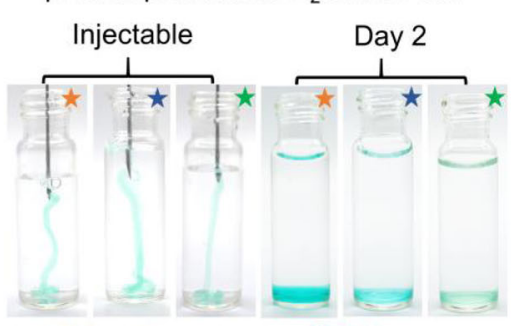

$\star \mathrm{pH} 2 \star \mathrm{DI}$ water $\star \mathrm{pH} 7.4$

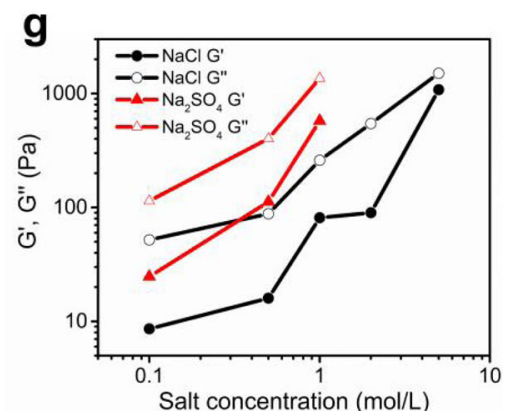

Fig. 1 Core-shell nanoparticles assemble into noncomplex coacervate to adapt to the harsh gastrointestinal (GI) tract environment. a Schematic illustration of harsh environmental conditions in the peristaltic GI tract. b Our noncomplex nanoparticle-assembled (NPA) coacervate compared with the conventional $\mathrm{pH}$ - and salt-dependent complex coacervate stabilized by electrostatic interactions between polyanions and polycations. $\mathbf{c}$ Driven by the gastrointestinal peristalsis, fluid NPA coacervate can effectively spread to coat and adhere on the large intestinal surface area via catechol-mediated wet bioadhesion. $\mathbf{d}$ The structure of as-prepared core-shell nanoparticles for fabricating NPA coacervates contains a hydrophobic core, hydrophilic PEG chains as the shell, and end groups of the PEG chains that provide physical interactions to induce nanoparticle assembly. The capacities of different nanoparticles $\left(\mathrm{NP}_{1}, \mathrm{NP}_{2}\right.$, and $\left.\mathrm{NP}_{3}\right)$ to form coacervates via nanoparticle assembly were examined. e The formation of NPA $\mathrm{A}_{2}$ coacervate (stained by Fast Green $\mathrm{FCF}$ ) via $\mathrm{NP}_{2}$ nanoparticle assembly can be visualized by fluid-fluid phase separation and further observed by TEM. $\mathbf{f}$ The liquid-like $\left(\mathrm{G}^{\prime}<\mathrm{G}^{\prime \prime}\right) \mathrm{NPA}_{2}$ coacervate (stained by Fast Green FCF) can be injected through a $21 \mathrm{G}$ needle and remained stable in buffers with a wide range of pH after 2 days. $\mathbf{g}$ Noncomplex $\mathrm{NPA}_{2}$ coacervate showed a salting-out effect, confirming that the formation of NPA coacervates should be attributed to hydrogen bonding-induced nanoparticle assembly rather than electrostatic interactions. Source data are provided as a Source Data file for Fig. 1g.

$5.0 \mathrm{M} \mathrm{NaCl}$, further confirming that the noncomplex NPA coacervation should be attributed to hydrogen bonding-induced nanoparticle assembly rather than electrostatic interactions. The rheological analysis confirmed the shear-thinning behavior of the NPA coacervates (Supplementary Figs. 8, 9). The viscosity of the $\mathrm{NPA}_{2}$ coacervate decreased quickly with an increasing shear rate from 0.1 to $20.0 \mathrm{~s}^{-1}$, thus insuring the easy injection of the $\mathrm{NPA}_{2}$ coacervate through a $21 \mathrm{G}$ needle. In contrast, the $\mathrm{NPA}_{3}$ coacervate demonstrated a much lower viscosity than that of the $\mathrm{NPA}_{2}$ coacervate, and this can be attributed to the weak interaction energy of $\pi-\pi$ interactions $\left(<10 \mathrm{~kJ} \mathrm{~mol}^{-1}\right)$ (Fig. 1d $)^{30}$.

Biocompatible and digestive enzyme-resistant $\mathrm{NPA}_{2}$ coacervate possesses reversible wet bioadhesion. Fluid $\mathrm{NPA}_{2}$ coacervate with a solid content of about $15 \mathrm{wt} \%$ flowed out from a tilted glass bottle and formed a durable adhesive coating on the medical nitrile glove (Fig. 2a and Supplementary Fig. 10). Furthermore, 


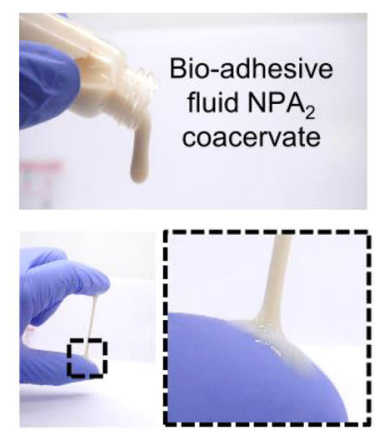

b

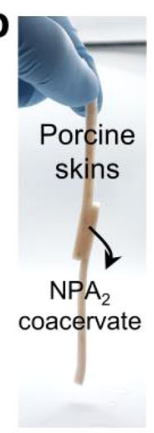

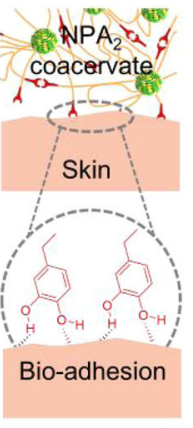
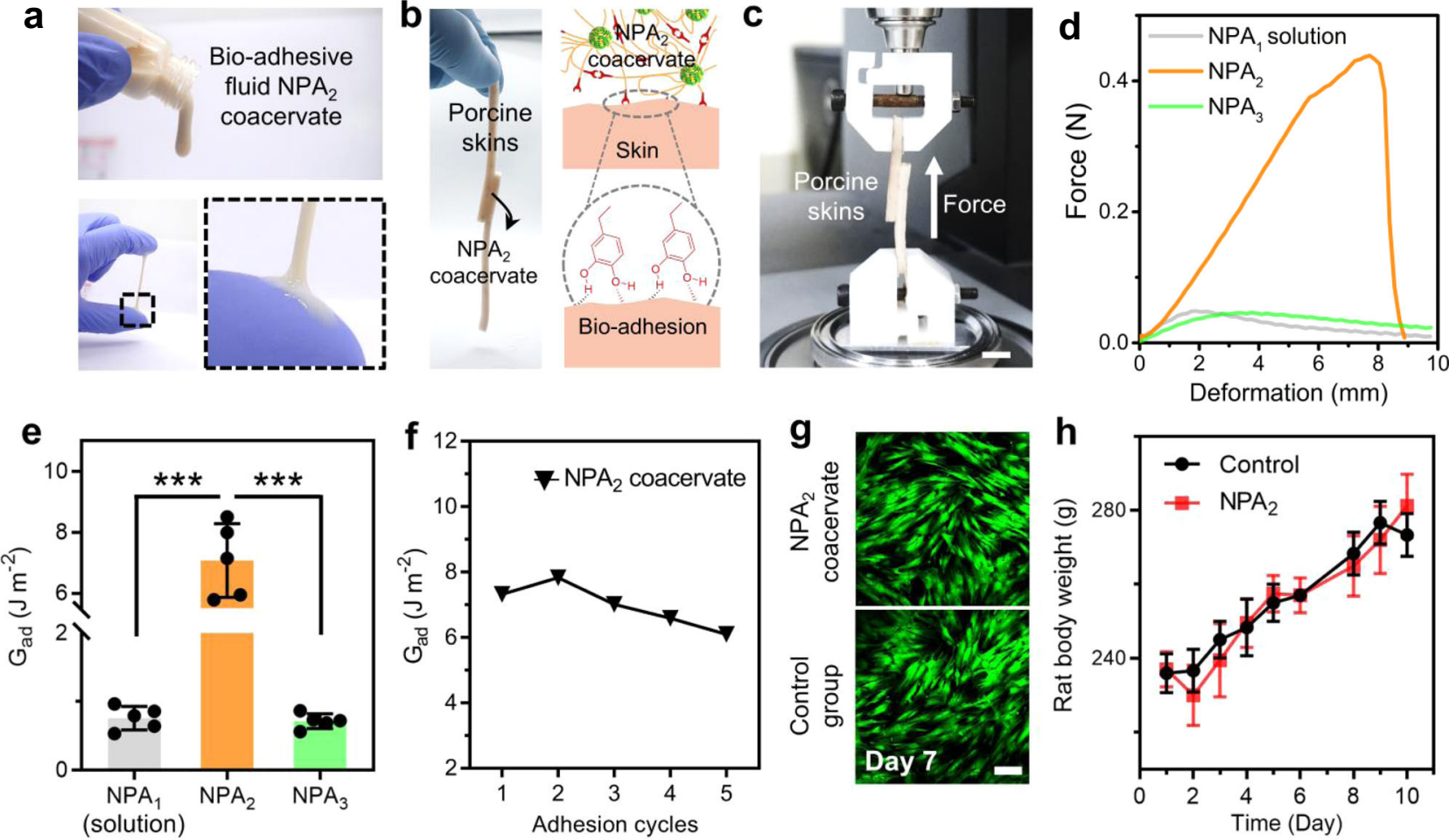

i
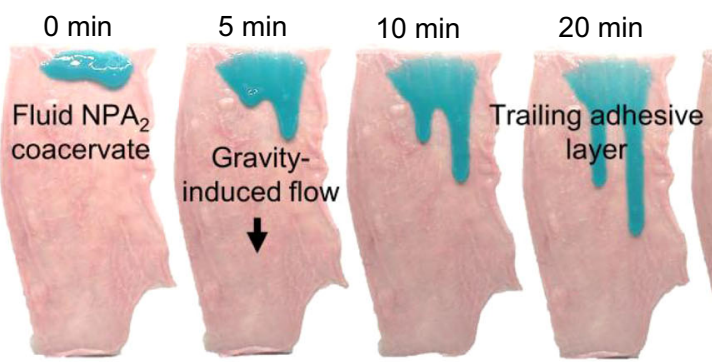

$40 \min$

Upright inner intestinal surface

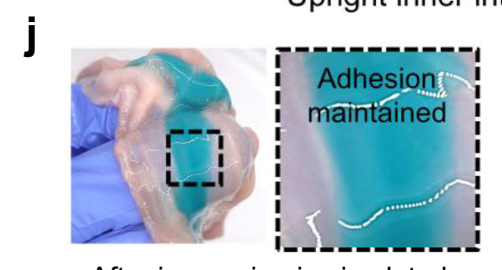

After immersion in simulated gastric fluid $(\mathbf{G a})$ for 2 hours

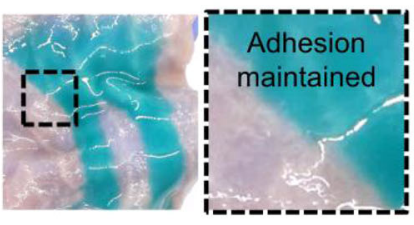

After immersion in simulated intestinal fluid (In) for 2 hours

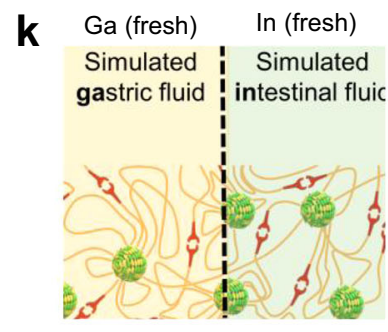

Day 3

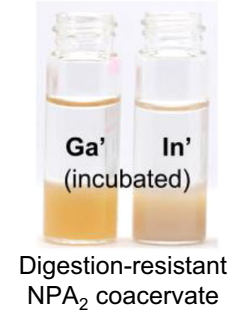

I No degraded $\mathrm{NPA}_{2}$ coacervate in $\mathrm{Ga}^{\prime} / \mathrm{ln}$

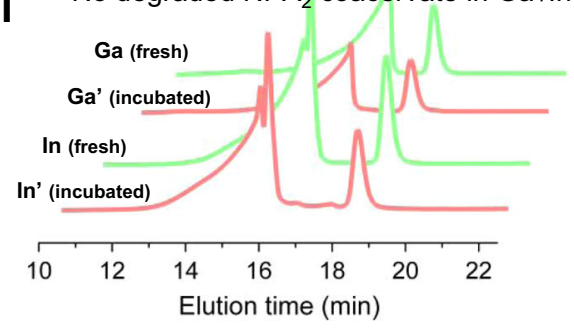

Fig. 2 Biocompatible and fluid $\mathbf{N P A}_{\mathbf{2}}$ coacervate is wet bioadhesive and resistant to digestive enzymes. a Fluid NPA $A_{2}$ coacervate formed a durable adhesive coating on the medical nitrile glove. $\mathbf{b} \mathrm{NPA}_{2}$ coacervate can glue two ribbons of porcine skin tissue together and hold the weight of the tissues. c Schematic of the lap-shear test to measure the adhesive energy $\left(G_{a d}\right)$. Scale bar: $10 \mathrm{~mm}$. $\mathbf{d}$, e The adhesion performance of NPA $\mathrm{A}_{2}$ coacervate was much better than that of the controls. NPA 2 coacervate showed adhesive energy $\left(\mathrm{G}_{\mathrm{ad}}\right)$ comparable with the previously reported values (around 2-10 J m ${ }^{-2}$ ) for nanoparticle-based adhesives and polymeric adhesives. $n=5$ independent lap-shear tests per group. $\mathbf{f}$ The hydrogen-bonding interactions derived from catechol groups ensure the stable and reversible adhesion energy level of $\mathrm{NPA}_{2}$ coacervate during the successive bonding/debonding cycles. $\mathbf{g}$ The viability staining of human mesenchymal stem cells (hMSCs) also suggested excellent cytocompatibility of the NPA $A_{2}$ coacervate. Scale bar: $200 \mu \mathrm{m}$. $\mathbf{h}$ No significant differences in body weights were observed between the rats receiving oral administration of NPA $A_{2}$ coacervate on days 1, 3, 5, 7, and 9, and untreated healthy rats during the 10-day toxicity evaluation. $n=3$ biologically independent rats in control group, $n=4$ biologically independent rats in $\mathrm{NPA}_{2}$ group. $\mathbf{i}$, $\mathbf{j}$ The fluid NPA 2 coacervate coating (stained by Fast Green FCF) can adhere to the fresh and wet mucosa, flow down slowly, and remain stable after soaking in simulated gastric fluid ( $\mathrm{Ga}$ ) and simulated intestinal fluid (In) at $37^{\circ} \mathrm{C}$ for $2 \mathrm{~h}$, respectively. Scale bar: $15 \mathrm{~mm}$. $\mathbf{k}, \mathbf{I} \mathrm{GPC}$ data of the fresh $(\mathrm{Ga}, \mathrm{In})$ and used simulated gastric or intestinal fluid $\left(\mathrm{Ga}^{\prime}, \mathrm{In}^{\prime}\right)$ incubated with NPA 2 coacervate. No degraded or dissolved NPA $\mathrm{A}_{2}$ coacervate components were found in the simulated gastric or intestinal fluid, respectively. Data were presented as mean \pm SD. ${ }^{\star \star \star} p<0.001$ (Ordinary one-way ANOVA). Source data are provided as a Source Data file for Fig. 2d-f, h. 
the catechol-mediated wet bioadhesion of $\mathrm{NPA}_{2}$ coacervate was sufficiently strong to glue two ribbons of the porcine skin tissue together and hold the tissue weight (Fig. 2b) ${ }^{15}$. The adhesive energy $\left(\mathrm{G}_{\mathrm{ad}}\right)$ of $\mathrm{NPA}_{2}$ coacervate was estimated to be $\sim 7.07 \mathrm{~J} \mathrm{~m}^{-2}$, similar to the previously reported values (around $2-10 \mathrm{~J} \mathrm{~m}^{-2}$ ) for nanoparticle-based ${ }^{44}$ and polymeric adhesives (Fig. $2 \mathrm{c}, \mathrm{d}$, e and Supplementary Fig. 11) ${ }^{45,46}$. The $\mathrm{NPA}_{2}$ coacervate-bonded porcine skin joints can withstand an applied force five times greater than that of the homolog control groups $\left(\mathrm{NPA}_{1}\right.$ nanoparticle solution and $\mathrm{NPA}_{3}$ coacervate). Furthermore, the peak adhesion force of the $\mathrm{NPA}_{2}$ coacervate was achieved at a much larger deformation, indicating the capability to effectively dissipate a large amount of energy under a mechanical challenge (Fig. 2d). The low adhesion energy of the control homologs can be attributed to the weak bulk cohesion $\left(\mathrm{NPA}_{1}\right.$ solution) or interfacial adhesion ( $\mathrm{NPA}_{3}$ coacervate), respectively. Therefore, although the $\pi-\pi$ interaction can facilitate coacervation, the negligible bioadhesion of the $\mathrm{NPA}_{3}$ coacervate makes it less suitable for drug delivery in the intestinal tract. Furthermore, reversible hydrogen bonding interactions derived from the catechol groups ensure the stable adhesion energy level of the $\mathrm{NPA}_{2}$ coacervate during the bonding/debonding cycles (Fig. $2 \mathrm{f}$ ). Consistent with the observed bioadhesion on porcine skins, the $\mathrm{NPA}_{2}$ coacervate showed decent mucoadhesion $\left(2.74 \mathrm{~J} \mathrm{~m}^{-2}\right)$ on the luminal surface of porcine intestines (Supplementary Fig. 12), which can be attributed to the physical interactions between the catechol/PEG structure of coacervate and glycosylated mucins ${ }^{47,48}$.

To evaluate the safety of oral administration, we first tested the cytotoxicity of our $\mathrm{NPA}_{2}$ coacervate by incubating it with human mesenchymal stem cells (hMSCs). Viability staining and cell metabolic assays (MTT) of hMSCs incubated with $\mathrm{NPA}_{2}$ coacervate for 7 days suggested excellent cytocompatibility of the $\mathrm{NPA}_{2}$ coacervates (Fig. $2 \mathrm{~g}$ and Supplementary Fig. 13). Furthermore, Sprague Dawley (SD) rats were gavaged with $\mathrm{NPA}_{2}$ coacervate ( $4 \mathrm{~g}$ per kg rat) every other day for 10 days. During the 10 -day exposure period, the rats receiving oral administration of coacervate did not develop any abnormal symptoms such as diarrhea and showed no significant weight change compared with the untreated healthy rats, further confirming the minimal toxicity of NPA coacervates (Fig. 2h).

We next investigated the influence of the physical peristalsis and chemical environment (gastric acid and intestinal fluid) of the GI tract on $\mathrm{NPA}_{2}$ coacervate coating by using simulated ex vivo experiments. When deposited on the upright intestinal mucosa surface, the fluid $\mathrm{NPA}_{2}$ coacervate can adhere to the fresh and wet mucosa and steadily flow down driven by gravity, leaving a trailing adhesive coating layer (Fig. 2i). After soaking the $\mathrm{NPA}_{2}$ coacervate-coated intestinal mucosa tissues in simulated gastric fluid $(\mathrm{Ga})$ or simulated intestinal fluid (In) at $37^{\circ} \mathrm{C}$ for $2 \mathrm{~h}$, respectively, the adherent coacervate coatings remained undiluted and maintained adhesion on the mucosa surface (Fig. $2 \mathrm{j}$ and Supplementary Fig. 14). The $\mathrm{NPA}_{2}$ coacervate also remained largely stable in the $25 \mathrm{mg} / \mathrm{ml}$ pig bile salt or under the high shear strain (up to $1000 \%$ ) despite a slight dip in shear moduli toward the high shear range (Supplementary Figs. 15, 16). In addition, the digestive enzyme-resistant polymeric structure of the $\mathrm{NPA}_{2}$ coacervate was further confirmed by incubating $\mathrm{NPA}_{2}$ coacervate in simulated gastric fluid (Ga) and simulated intestinal fluid (In) at $37^{\circ} \mathrm{C}$ for 3 days, respectively (Fig. 2k). GPC analysis showed that no degraded or dissolved $\mathrm{NPA}_{2}$ coacervate components were found in the simulated body fluids $\left(\mathrm{Ga}^{\prime}, \mathrm{In}\right.$ ') after 3 days of incubation (Fig. 2l). It is noted that the nontoxic, biologically, and chemically inert polyurethanes are widely used in clinical practices $^{49,50}$, and a polyurethane-based surgical adhesive $\left(\right.$ TissuGlu $^{\oplus}$ ) has been approved by United States Food and Drug Administration (FDA) for various clinical applications.
Therefore, we believe that the stable polyurethane network of $\mathrm{NPA}_{2}$ coacervate helps establish the feasibility for the following in vivo evaluations. Besides, our laboratory-scale synthesis yielded $\sim 300 \mathrm{ml}$ of $\mathrm{NPA}_{2}$ coacervates per batch and showed stable batchto-batch reproducibility, indicating the promising potential for large-scale production of $\mathrm{NPA}_{2}$ coacervates.

$\mathrm{NPA}_{2}$ coacervate optimizes drug release with extended GI residence time in vivo. We believe that the water-immiscible, bioadhesive, and liquid nature of our noncomplex $\mathrm{NPA}_{2}$ coacervate can adapt to the complex motility, fluid content, and sharp $\mathrm{pH}$ variation within the GI tract, and establish a large-area durable coating on the intestinal mucosa to ensure sufficient intestinal retention for sustained drug release. To further test this hypothesis, the capability of NPA coacervates as bioadhesive coatings on the gastrointestinal mucosa was further evaluated in vivo (Fig. 3a). Sprague Dawley (SD) rats with unrestricted access to water and standard laboratory diet received a single oral gavage of $1.0 \mathrm{ml} \mathrm{NPA}_{2}$ or $\mathrm{NPA}_{3}$ coacervate (modified with a Cy7 tag, a near-infrared fluorescent dye) and were sacrificed after 0,6 , 24 , or $48 \mathrm{~h}$ to evaluate the fluorescence retention in the harvested GI tract (Fig. 3b). In agreement with the in vitro bioadhesive property, the $\mathrm{NPA}_{2}$ coacervate adhered to the rat GI tract for at least $48 \mathrm{~h}$ as evidenced by the intense fluorescence signal, whereas the $\mathrm{NPA}_{3}$ coacervate had limited retention in the GI tract after 1 day (Fig. 3b). Normal gastric emptying takes place within $2 \mathrm{~h}$, and colonic arrival occurs after $5 \mathrm{~h}^{51}$. Therefore, the in vivo intestinal retention experiments demonstrated the prolonged retention of our $\mathrm{NPA}_{2}$ coacervate in the GI tract.

We further evaluated the efficacy of the $\mathrm{NPA}_{2}$ coacervate to mediate sustained drug release both in vivo and in vitro. We utilized Dex-P, a water-soluble sodium phosphate salt of dexamethasone (Dex), as a model anti-inflammatory drug ${ }^{52}$. The $\mathrm{NPA}_{2}$ coacervate showed a high Dex-P encapsulation efficiency (the amount of Dex-P loaded in the $\mathrm{NPA}_{2}$ coacervate divided by the total Dex-P input) of $\sim 42 \%$ (Fig. $3 \mathrm{c}$ and Supplementary Fig. 17). The partition coefficient (K), which was defined as the ratio between the concentration of Dex-P in the $\mathrm{NPA}_{2}$ coacervate and the supernatant Dex-P concentration, was around 3.62 (Fig. 3d). In addition to the excellent drug loading capacity, our $\mathrm{NPA}_{2}$ coacervate showed prolonged release of preloaded Dex-P under both in vitro (Fig. 3e) and in vivo conditions (Fig. 3f). We next examined the drug release kinetics in vivo by monitoring the serum levels of Dex-P at selected time points after oral gavage of Dex-P-laden $\mathrm{NPA}_{2}$ coacervate or Dex$P$ aqueous solution (Fig. 3f). The serum concentration of Dex in $\mathrm{SD}$ rats spiked at $1 \mathrm{~h}$ after oral gavage of the Dex-P solution and then decreased rapidly (Dex-P/PBS). In contrast, the serum Dex concentration in the $\mathrm{SD}$ rats receiving oral gavage of Dex-Pladen $\mathrm{NPA}_{2}$ coacervate (Dex-P/NPA $)$ remained consistently at the lower therapeutic level for over $40 \mathrm{~h}$ (Fig. 3f). The total dosage of Dex-P administered in the two groups was the same. Therefore, our $\mathrm{NPA}_{2}$ coacervate can mediate sustained release and improve the pharmacokinetics of the preloaded drug in the SD rat model, thereby reducing dosing frequency and systemic drug exposure.

It is noted that ulcerative colitis is a chronic inflammatory disorder limited to the colonic and rectal mucosa ${ }^{53}$ and shows similar gastric and small intestinal transit time as that of healthy controls $^{54,55}$. Because absorption of the Dex-P, a water-soluble small molecule drug, mainly occurs in the small intestine in the absence of a drug carrier ${ }^{56}$, the serum Dex concentrations of DSSinduced colitic rats and healthy rats receiving the equivalent amount of Dex-P in PBS (Dex-P/PBS) were similar (Supplementary Fig. 18). We further tested the capability of $\mathrm{NPA}_{2}$ coacervates 

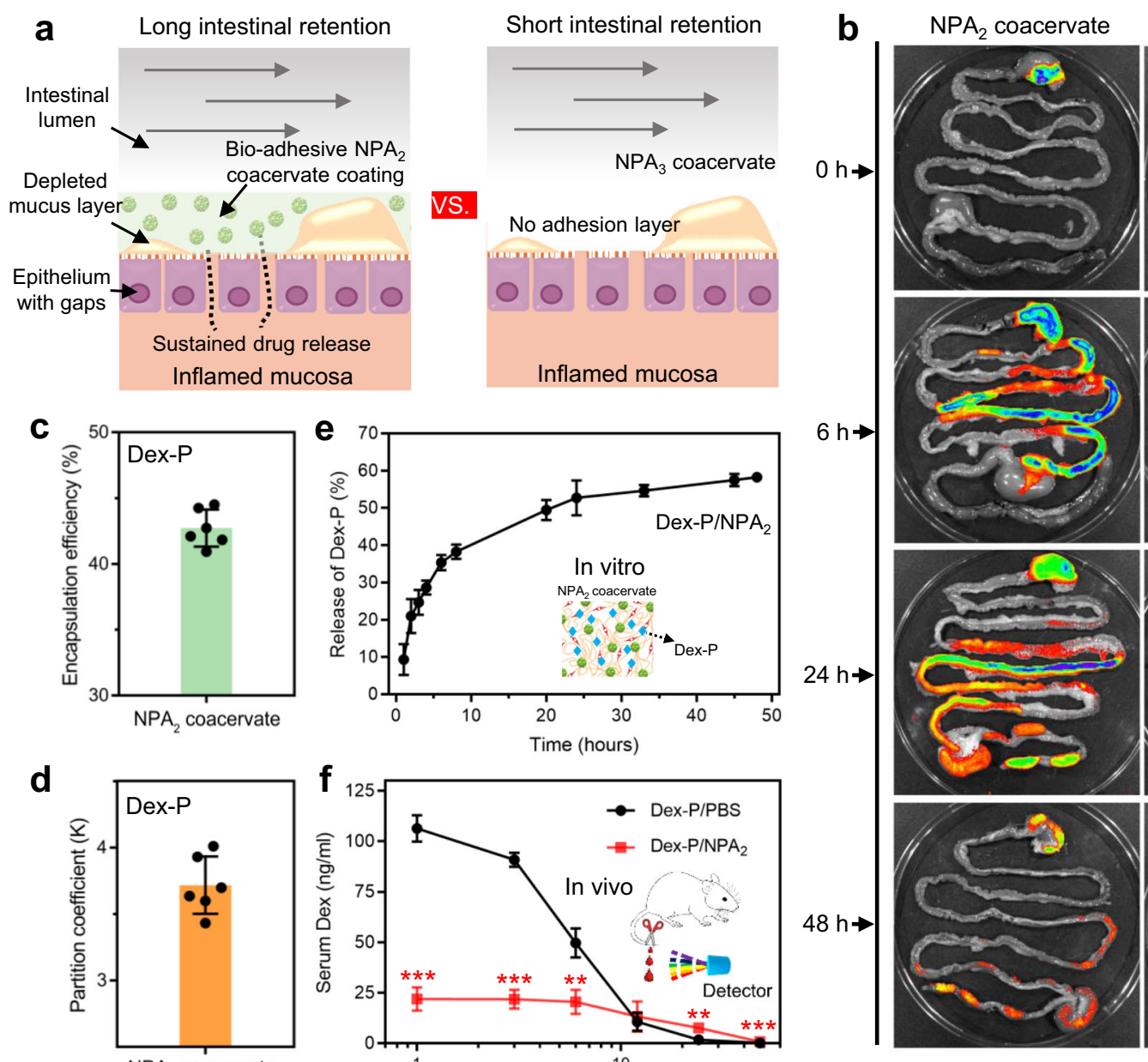

$\mathrm{NPA}_{3}$ coacervate
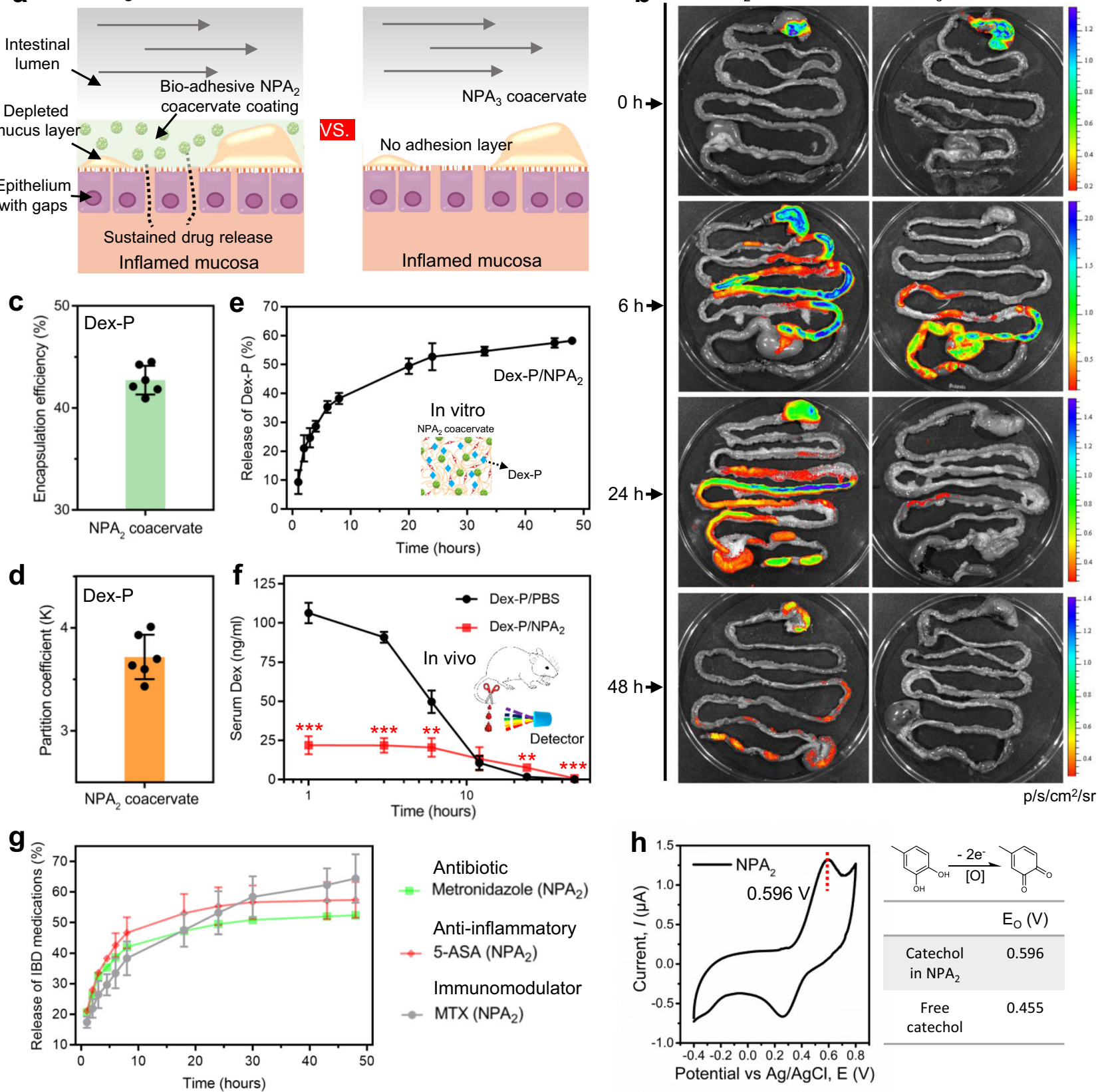

Fig. $3 \mathbf{N P A}_{\mathbf{2}}$ coacervate demonstrates prolonged retention and mediates sustained drug release in the $\mathbf{G I}$ tract. $\mathbf{a}$, $\mathbf{b}$ The bioadhesive NPA 2 coacervate delivered via oral gavage adhered to the GI tract of SD rats for at least 2 days, whereas the control nonadhesive NPA $\mathrm{A}_{3}$ coacervate showed short retention in the GI tract. c, d NPA 2 coacervate has a high Dex-P encapsulation efficiency because of the hydrophobic component of its structure. $n=6$ independent encapsulation tests. e NPA ${ }_{2}$ coacervate mediated sustained release of preloaded Dex-P over several days in vitro. $\mathbf{f}$ Dex-P-laden NPA 2 coacervate delivered by oral gavage better sustained the serum Dex concentration at the lower therapeutic level compared with that of the oral gavage of Dex- $P$ aqueous solution (Dex-P/PBS) in vivo. $n=3$ biologically independent rats per group. $\mathbf{g}$, $\mathbf{h}$ The condensed hydrophobic environment of NPA $A_{2}$ coacervate as revealed by an increased catechol oxidation potential $\left(E_{0}\right)$ of $0.596 \mathrm{~V}$, facilitated the sustained release of a wide array of water-soluble small-molecular drugs including antibiotic metronidazole, anti-inflammatory 5-aminosalicylic acid (5-ASA), and immunoregulatory methotrexate disodium salt (MTX). Data were presented as mean \pm SD. ${ }^{\star} p<0.05,{ }^{\star \star} p<0.01,{ }^{\star \star \star} p<0.001$ (two-tailed Student's $t$-test). Source data are provided as a Source Data file for Fig. 3c-h.

as bioadhesive coatings to mediate the sustained release of drugs in a DSS-induced colitic rat model. The observed retention of fluorescently-label coacervate in the harvested GI tract revealed that the $\mathrm{NPA}_{2}$ coacervate can adhere to the GI tract of colitic rats for at least $48 \mathrm{~h}$, thus confirming the similar retention time of our $\mathrm{NPA}_{2}$ coacervate in the GI tract of colitic rats compared with that of the healthy rats (Supplementary Fig. 19), and this explained the observed similar release and uptake kinetics of coacervate-delivered
Dex-P (as indicated by serum Dex concentration) between the colitic and healthy rats (Supplementary Figs. 19, 20).

To demonstrate the versatility of $\mathrm{NPA}_{2}$ coacervate to mediate sustained drug release, other first-line small molecule drugs used to treat IBD including antibiotic metronidazole, antiinflammatory 5-aminosalicylic acid (5-ASA), and immunoregulatory methotrexate disodium salt (MTX) were also encapsulated into the $\mathrm{NPA}_{2}$ coacervate to investigate the release kinetics 
(Fig. 3g). The $\mathrm{NPA}_{2}$ coacervate showed high encapsulation efficiency and prolonged release kinetics of these drugs (Supplementary Fig. 17). In addition, the dried drug-laden $\mathrm{NPA}_{2}$ coacervate that is desirable for oral administration could be prepared via lyophilization, and simply adding dried drugladen $\mathrm{NPA}_{2}$ coacervate into simulated gastric fluid or water could realize the rehydration to form fluid $\mathrm{NPA}_{2}$ coacervate with sustained-release kinetics of diverse drugs similar to the freshly prepared drug-laden $\mathrm{NPA}_{2}$ coacervate before lyophilization (Supplementary Fig. 21).

We hypothesized that the capability of $\mathrm{NPA}_{2}$ coacervates to mediate the sustained release of water-soluble small molecules such as Dex-P can be ascribed to the relatively hydrophobic environment within the $\mathrm{NPA}_{2}$ coacervate. We confirmed the above hypothesis by examining the cyclic voltammetry (CV) curve of the $\mathrm{NPA}_{2}$ coacervate (Fig. $3 \mathrm{~h}$ ). Our $\mathrm{NPA}_{2}$ coacervate showed a catechol oxidation potential $\left(\mathrm{E}_{0}\right)$ of $0.596 \mathrm{~V}$ in deionized water, which was significantly higher than the $E_{0}$ of soluble small molecule 4 -methylcatechol $(0.455 \mathrm{~V})^{57}$. The high catechol oxidation potential suggests that the $\mathrm{NPA}_{2}$ coacervate with a high mass ratio of hydrophobic cores provides a highly stabilizing hydrophobic environment against catechol oxidation by shielding from the aqueous solvent ${ }^{58}$, thus facilitating the efficient loading and sustained release of a wide array of drugs.

Dex-P-laden $\mathrm{NPA}_{2}$ coacervate shows potent therapeutic efficacy against acute colitis in a rodent model. We next evaluated the therapeutic efficacy of the Dex-P-laden $\mathrm{NPA}_{2}$ coacervate in the rat model of dextran sulfate sodium (DSS)-induced colitis (Fig. 4a). SD rats weighing around $250 \mathrm{~g}$ were given $4.5 \%$ DSS in drinking water for 7 days to develop acute colitis ${ }^{25}$. Clinical manifestations of the colitis, such as severe rectal bleeding, watery diarrhea, and colonic edema were observed after 7 days (Supplementary Fig. 22). After successfully establishing the colitis model in rats, colitic $\mathrm{SD}$ rats received oral gavages of Dex-P-laden $\mathrm{NPA}_{2}$ coacervates (Dex-P/NPA $)$ or the equivalent amount of Dex-P in PBS (Dex-P/PBS) on days 1, 3, and 5 (Fig. 4b). Untreated colitic SD rats were used as the negative control. All SD rats were allowed unrestricted access to water and standard laboratory diet before and after oral gavage and sacrificed on day 7 for further evaluation of colon weight and length, histological severity, IBD-associated colonic myeloperoxidase (MPO)-activity, mRNA levels, and protein expressions of tight junction-associated proteins (ZO-1 and occludin-1) and pro-inflammatory cytokines, such as interleukin $I L-1 \beta$ and tumor necrosis factor (TNF) in the distal colon.

Our results demonstrated the significant therapeutic efficacy of Dex-P/NPA 2 treatment against DSS-induced acute colitis. Dex-Pladen $\mathrm{NPA}_{2}$ coacervate significantly alleviated colonic edema and diarrhea caused by DSS-induced acute colitis (Fig. 4c). Relieved edema in colitic SD rats receiving Dex- $/ \mathrm{NPA}_{2}$ was further confirmed by the lower colon (cecum) weight/length ratio (Fig. 4d). Representative images of hematoxylin and eosin (H\&E) staining demonstrated significantly reduced histological inflammation in the colitic SD rats receiving Dex-P/NPA histological damages, such as the compromised integrity of the mucosal epithelial lining, decrease in villus height and crypt depth, interstitial edema, and inflammatory infiltration were observed in untreated colitic SD rats (Control) or rats treated with the equivalent amount of Dex-P solution in PBS (Dex-P/ PBS, Fig. 4e). Furthermore, histopathology scoring of H\&Estained tissue sections was used to evaluate the severity of colonic histological damage in a blinded fashion by a trained pathologist. Disease severity in colitic $\mathrm{SD}$ rats receiving Dex-P/NPA decreased significantly (mean histopathology score, 0.500) compared with colitic SD rats in the Dex-P/PBS group (mean histopathology score, 1.917) and the untreated control group (mean histopathology score, 3.000). Histopathology scores of colitic SD rats in the Dex-P/PBS group were not significantly different from the untreated control group $(P=0.056$, Fig. $4 \mathrm{f})$.

Colonic MPO activity in colitic SD rats receiving Dex- $\mathrm{P} / \mathrm{NPA}_{2}$ was also significantly reduced compared with the untreated control group (Fig. 4g) ${ }^{59}$. Although we also observed a reduction of MPO activity in the Dex-P/PBS group due to the therapeutic activity of Dex-P against IBD, the high serum Dex level associated with such administration of Dex-P aqueous solution indicates an increased risk of complications related to severe systemic drug exposure (Fig. 3f). We have also tested the therapeutic efficacy of $\mathrm{NPA}_{2}$ alone to treat colitic rats via oral gavage on days 1,3 , and 5 and then analyzed the MPO activity and histopathology score of colon tissues on day 7 (Supplementary Fig. 23). The therapeutic efficacy of treatment with $\mathrm{NPA}_{2}$ alone did not show a significant difference compared with that of the non-treated colitic rats (Control) as evidenced by the similar MPO activity, histopathology score, and H\&E staining, thus confirming that the $\mathrm{NPA}_{2}$ coacervate only served as a drug delivery vehicle and did not mitigate the colitic symptoms. In addition, colitic SD rats receiving Dex-P/NPA 2 recovered the expression of tight junction-associated proteins including ZO-1 and occludin- 1 and showed significantly reduced local levels of IL-1 $\beta$, IL-6, and TNF compared with the untreated group and Dex-P/PBS group (Fig. 4h, i and Supplementary Figs. 24, 25). Taken together, oral delivery of Dex-P encapsulated in $\mathrm{NPA}_{2}$ coacervate to colitic SD rats showed significantly enhanced therapeutic outcomes and reduced systemic exposure than administering the equivalent amount of Dex-P in an aqueous solution (Supplementary Fig. 26).

Dex-P/NPA 2 regulates innate immune responses and restores the gut microbiota. Macrophage activation can be broadly classified into the pro-inflammatory M1 polarization or antiinflammatory M2 polarization ${ }^{60}$, and macrophages play a key role in the maintenance of mucosal homeostasis by secreting many cytokines ${ }^{61}$. Therefore, we next investigated the intestinal immune responses of colitic SD rats by analyzing macrophage polarizations in the harvested colon tissues from colitic rats receiving Dex-P/NPA 2 or Dex-P/PBS and untreated colitic $\mathrm{SD}$ rats (Control) on day 5 (Fig. 5a). Many studies have shown that dexamethasone (Dex), a corticosteroid, can promote antiinflammatory M2 macrophage polarization and suppress proinflammatory M1 polarization ${ }^{62-64}$, thus enhancing the secretion of anti-inflammatory cytokines, such as IL-1065. Immunohistochemistry staining against CD206 (M2 marker) or iNOS (M1 marker) showed increasing M2 macrophage polarization in the colitic SD rats receiving Dex-P/NPA 2 (Fig. $5 b$ and Supplementary Fig. 27). Furthermore, Dex- $/ / \mathrm{NPA}_{2}$ treatment significantly reduced the local levels of pro-inflammatory IL- $1 \beta$ and IL- 6 released by M1 macrophages (Fig. 5e) and increased the level of anti-inflammatory IL-10 (Fig. 5f) ${ }^{66}$. Taken together, oral administration of Dex-P/NPA 2 to colitic SD rats promoted the M2 polarization of intestinal macrophages ${ }^{61}$ and mediated strong anti-inflammatory effects against acute colitis, thereby creating a favorable immune microenvironment to promote colon repair and regeneration (Fig. $5 \mathrm{c}, \mathrm{d}$ ).

Dysbiosis of gut microbiota, which is implicated in various inflammatory and immune diseases ${ }^{67}$, plays a key role in the pathogenesis of IBD, while the chronic inflammation caused by IBD, in turn, promotes dysbiosis of gut microbiota ${ }^{68}$. Therefore, restoring gut microbiota and recovery of gut immune homeostasis can complement each other in the successful treatment of acute colitis. Although orally administered Dex-P may not 


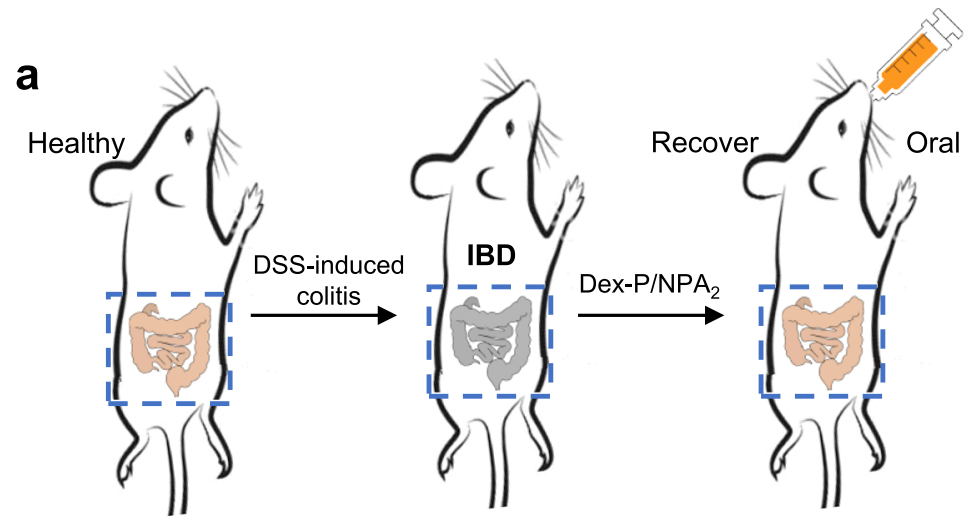

C Colonic damage at Day 7

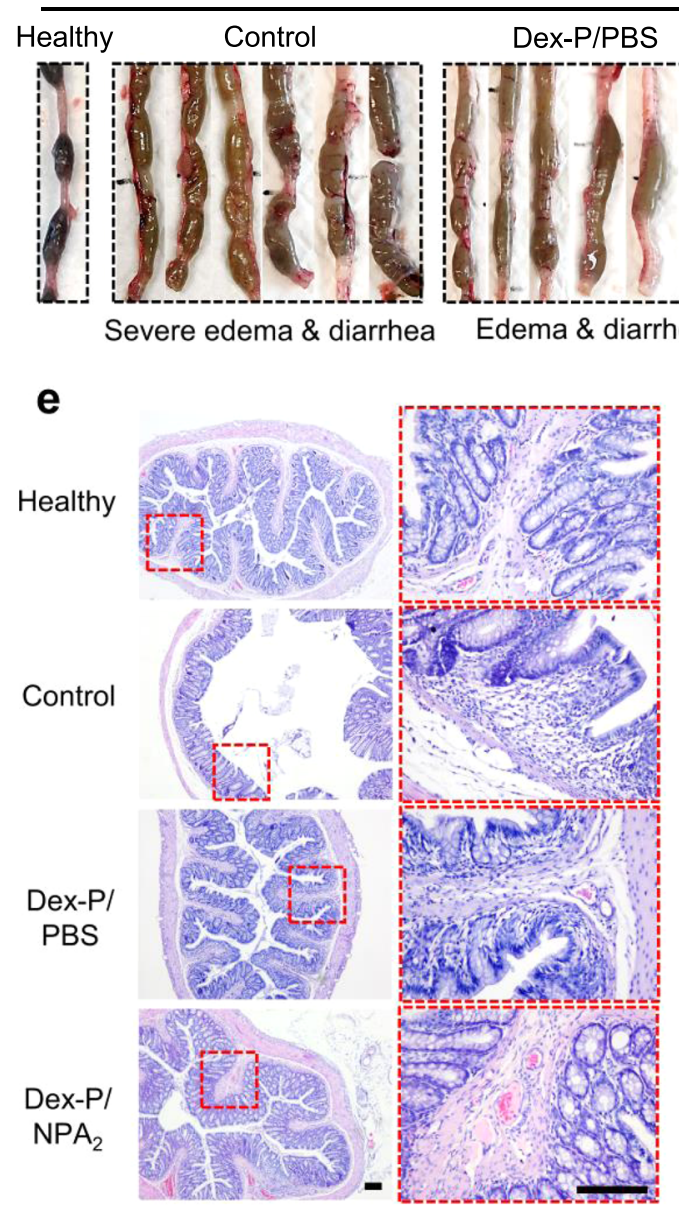

b

Dex-P/PBS, Dex-P/NPA 2

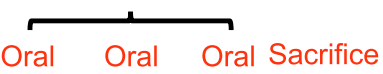

Day 1 Day 3 Day 5 Day 7

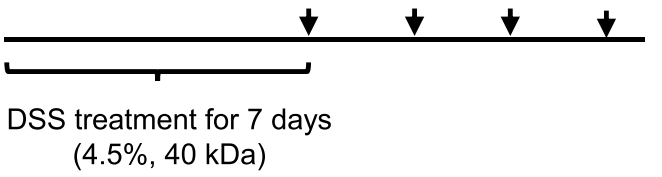

d

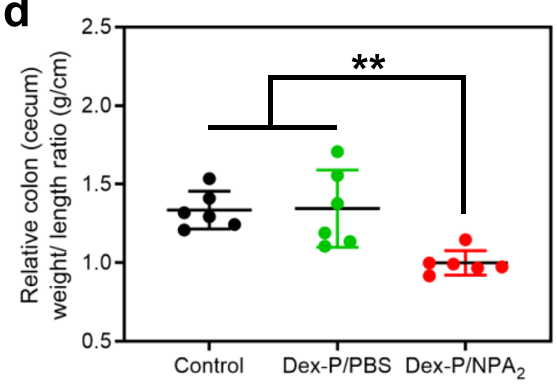

$\mathbf{f}$

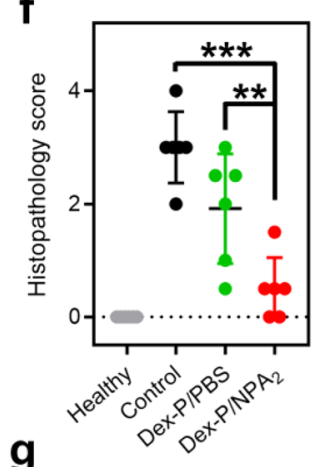

h
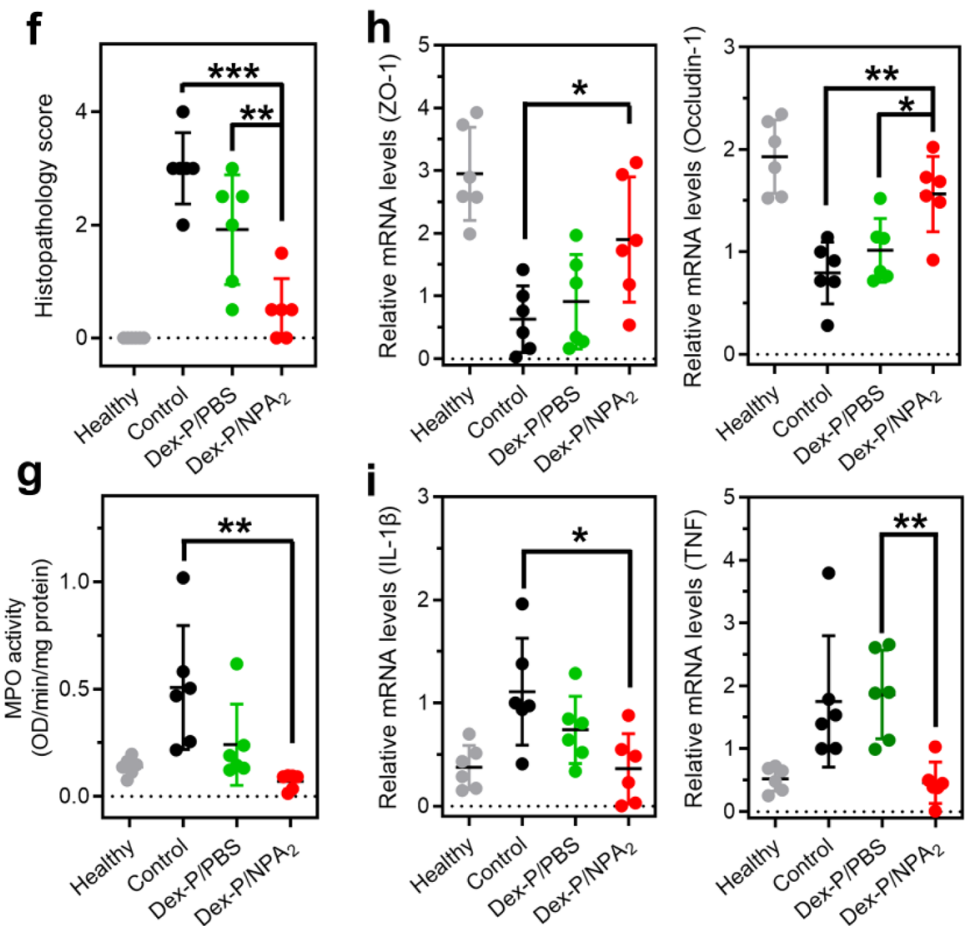

Fig. 4 Dex-P-laden $\mathbf{N P A}_{\mathbf{2}}$ coacervate demonstrates enhanced therapeutic efficacy in a rat model of DSS-induced colitis. a, $\mathbf{b}$ SD rats were given $4.5 \%$ DSS in drinking water to induce acute colitis. The colitic rats received oral gavages of Dex-P-laden NPA 2 coacervates (Dex-P/NPA $)$ or the equivalent amount of Dex-P in PBS (Dex-P/PBS) on days 1, 3, and 5. Untreated colitic SD rats were used as the negative control (Control). All SD rats were sacrificed on day 7. c, d Colonic edema and diarrhea caused by DSS-induced colitis in SD rats receiving Dex-P/NPA 2 were significantly relieved compared with that of the untreated colitic SD rats (Control) and colitic SD rats receiving Dex-P in PBS (Dex-P/PBS). $n=6$ biologically independent rats per group. Scale bar: $10 \mathrm{~mm}$. e Representative images of H\&E staining demonstrated that histological inflammation was diminished in the colitic $\mathrm{SD}$ rats receiving Dex-P/NPA , while histological damages were observed in untreated colitic SD rats (Control) or colitic SD rats receiving Dex-P/PBS. Scale bar: $150 \mu \mathrm{m}$. f-i On day 7, colon tissues were analyzed for histopathology score $\mathbf{f}$, MPO activity $\mathbf{g}$, mRNA levels of tight junction-associated proteins including ZO- 1 and occludin- $1 \mathbf{h}$, and pro-inflammatory cytokines including interleukin $I L-1 \beta$ and TNF $\mathbf{i}$. Data were presented as mean \pm SD. $n=6$ biologically independent rats per group. ${ }^{\star} p<0.05,{ }^{\star \star} p<0.01,{ }^{\star \star \star} p<0.001$ (Ordinary one-way ANOVA). Source data are provided as a Source Data file for Fig. 4d, f-i. 

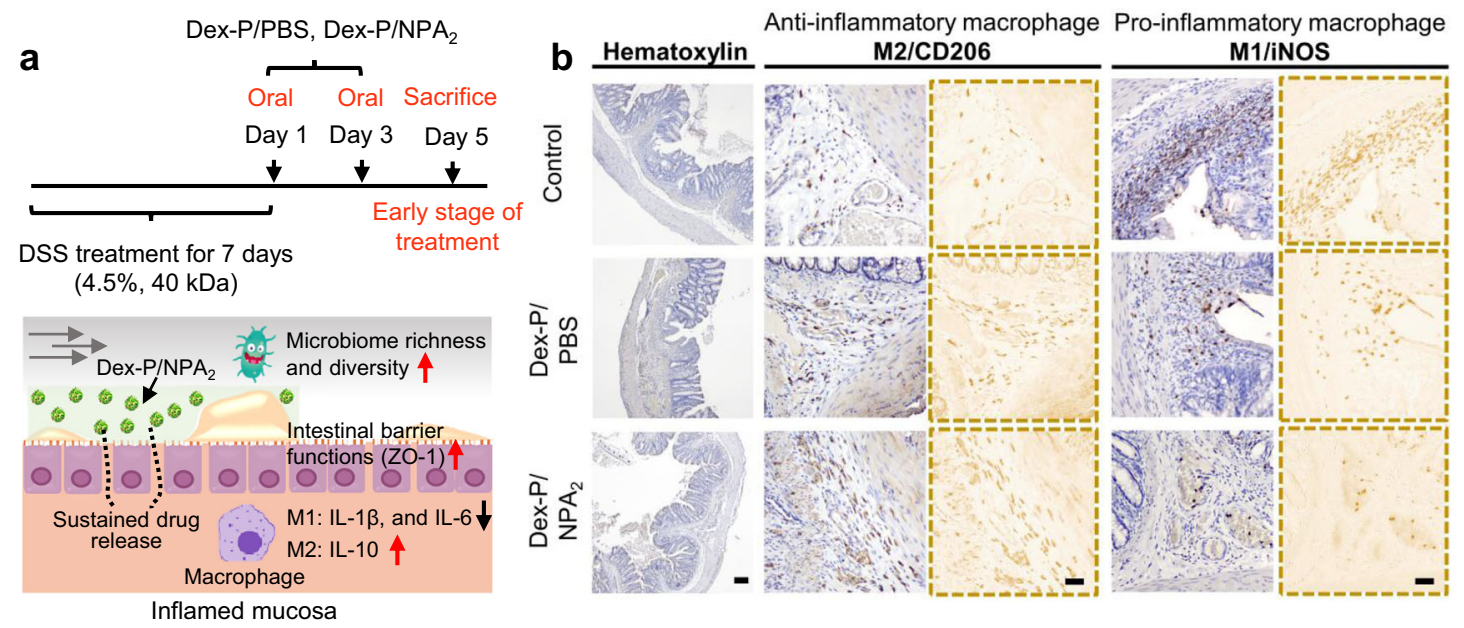

Dex-P/NPA ${ }_{2}$ promotes rapid recovery of acute colitis
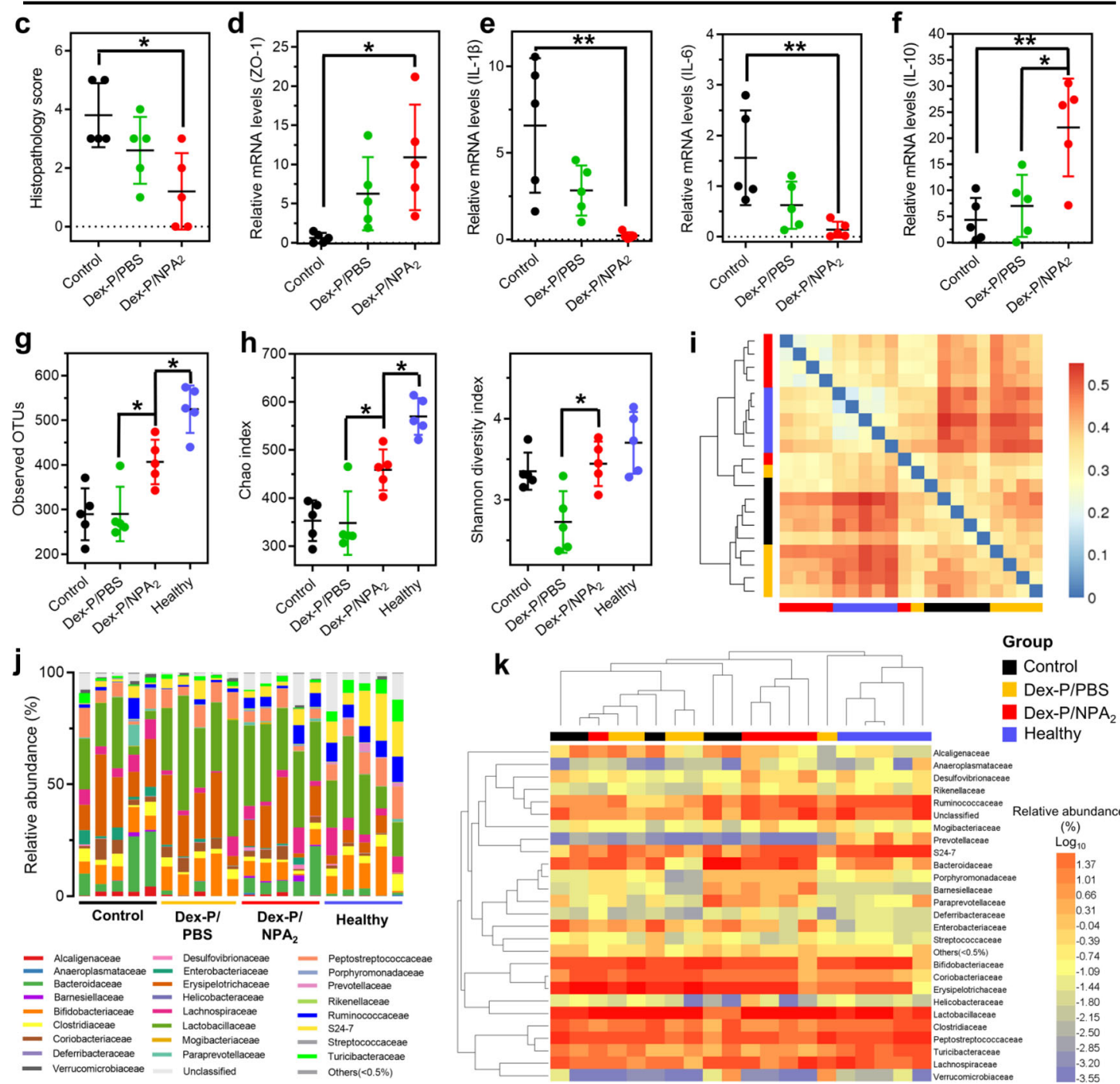

directly modulate the gut microbiota ${ }^{69}$, we expected the enhanced gut immune microenvironment by Dex- $\mathrm{P} / \mathrm{NPA}_{2}$ treatment can positively regulate the gut microbiota. Analyses of fecal samples collected from colitic SD rats on day 5 by sequencing the V4 region of the $16 \mathrm{~S}$ ribosomal ribonucleic acid (rRNA) gene showed that Dex-P/NPA 2 treatment indeed increased the bacterial richness (observed operational taxonomic units, OTUs) and diversity (Chao and Shannon indices) in colitic SD rats (Fig. $5 \mathrm{~g}, \mathrm{~h}$ ). In addition, a heatmap of $\beta$-diversity distance distribution was generated, and samples with similar $\beta$-diversity were clustered to reflect the similar compositions of gut microbiota (Fig. 5i). The $\beta$-diversities between colitic SD rats receiving Dex-P/NPA 2 and healthy SD rats were clustered more closely compared with colitic SD rats in the Dex-P/PBS group and 
Fig. 5 Dex-P/NPA $\mathbf{A}_{\mathbf{2}}$ treatment regulates the innate immune responses and restores the gut microbiota. a-f On day 5, colitic SD rats receiving Dex- $\mathrm{P} /$ $\mathrm{NPA}_{2}$, or Dex-P/PBS, and untreated colitic SD rats (Control) were sacrificed, and colon tissues were analyzed for macrophage polarization. b Immunohistochemical staining against key macrophage M1/M2 polarization markers iNOS/CD206, $\mathbf{c}$ histopathology score, $\mathbf{d}$ mRNA levels of tight junction-associated protein ZO-1, e pro-inflammatory cytokines including interleukin IL-1 $\beta$ and IL-6 released by M1 macrophages, and $\mathbf{f}$ antiinflammatory cytokine IL-10 released by M2 macrophages. Scale bar for hematoxylin staining, $200 \mu \mathrm{m}$. Scale bar for immunohistochemistry staining, $50 \mu \mathrm{m} . n=5$ biologically independent rats per group. g-k Fecal samples collected on day 5 from colitic SD rats were analyzed for gut microbiota by sequencing the $\mathrm{V} 4$ region of the $16 \mathrm{~S}$ rRNA gene. Dex-P/NPA 2 treatment increased $\mathbf{g}$ bacterial richness (observed operational taxonomic units, OTUs), $\mathbf{h}$ Chao diversity, and $\mathbf{h}$ Shannon diversity in colitic SD rats compared with colitic SD rats in the Dex-P/PBS group and the untreated colitic rats (Control). $n=5$ biologically independent rats per group. i A clustered heatmap of UniFrac values for measuring gut microbiota $\beta$-diversity illustrated that colitic SD rats receiving Dex-P/NPA 2 and healthy SD rats were clustered more closely, suggesting more similar bacterial compositions. The color of the square shows the distance of evolution between each two samples. The range of blue to red corresponds to close to far distance, and the bigger index means the greater differences between samples. $\mathbf{j}$ Taxonomic bacterial distribution histogram and $\mathbf{k}$ clustered heatmap based on the relative abundance $\left(\log _{10}\right)$ of the gut microbiota at the family level are presented. The upper longitudinal clustering indicates the similarity of gut microbiota among individual SD rats. The closer distance and shorter branch length indicate more similar gut microbiota between the SD rats. Data were presented as mean \pm SD. ${ }^{\star} p<0.05,{ }^{\star \star} p<0.01,{ }^{\star \star \star} p<0.001$ (Ordinary one-way ANOVA). Source data are provided as a Source Data file for Fig. $5 c-k$.

the untreated control group (Control), thus suggesting the enhanced recovery of gut microbiota in colitic SD rats by Dex$\mathrm{P} / \mathrm{NPA}_{2}$ treatment. This was further confirmed by the taxonomic bacterial distribution histogram and clustered heatmap based on the relative abundance of gut microbiota at the family level (Fig. 5j, k).

In conclusion, we have developed a simple yet highly efficient strategy for oral drug delivery by using a nanoparticle-assembled (NPA) bioadhesive noncomplex coacervate. The NPA coacervate exhibited: (1) $\mathrm{pH}$ and salt-independent stability under the complex environment within the GI tract; (2) effective coating of large intestinal surface and prolonged intestinal retention for several days; and (3) enhanced therapeutic efficacy for IBD and gut microbiota restoration with reduced systemic drug exposure via the controlled release of a loaded drug. When used for clinical treatments, these highly desired attributes of NPA coacervatebased oral drug delivery will potentially enhance patient compliance and acceptance and therefore the eventual therapeutic outcomes, especially compared with conventional therapies such as enema, subcutaneous and intravenous injection. Although we used Dex-P as a model drug to treat IBD in this study, our bioadhesive $\mathrm{NPA}_{2}$ coacervate can effectively deliver many other drugs, especially the water-soluble small molecule drugs, which are usually difficult to achieve sustained-release kinetics by using the existing oral drug delivery platforms. Therefore, we believe that the NPA coacervate-based drug delivery represents a promising approach to the treatment of numerous gastrointestinal diseases, including mucosal wound healing, gastrointestinal cancers, peptic ulcers, and viral infections.

\section{Methods \\ In situ assembly of as-prepared core-shell nanoparticles into NPA coacervate. The concentrated amphiphilic polymers composed of hydrophilic poly(ethylene glycol) (PEG) and hydrophobic segments in $20 \mathrm{ml}$ DMF ( 27 wt\%) were drop- ped into cold deionized water $(60 \mathrm{~mL})$ with $600 \mathrm{rpm}$ magnetic stirring to form the core-shell nanoparticles $\left(\mathrm{NP}_{1}, \mathrm{NP}_{2}\right.$, and $\left.\mathrm{NP}_{3}\right)$, respectively. The nanoparticle solutions then were dialyzed against deionized water (standard regenerated cellulose (RC) dialysis membrane with $3.5 \mathrm{kDa}$ cutoff, Spectrum Chemical) at room temperature for $24 \mathrm{~h}$ to form NPA coacervates via nanoparticle assembly. To determine the successful synthesis and the molecular weight of the hydrophobic segment of the amphiphilic polymers, $1.5 \mu \mathrm{L}$ of the hydrophobic segment in methanol $(1 \mathrm{mg} / \mathrm{ml})$ was premixed with $1.5 \mu \mathrm{L}$ of matrix solution (saturated $\alpha$-cyano-4-hydroxycinnamic acid, Sigma). Then this mixture was applied to a ground steel target that was ready for mass spectrometry. Finally, the analyses of air-dried samples were performed in an AutoFlex Speed LRF MALDI-TOF mass spectrometer (Bruker Daltonics, Germany) under reflector mode.}

In vitro biocompatibility test. Passage 4 hMSCs $\left(8 \times 10^{4}\right.$ cells/well, Lonza, Catalog \#: PT-2501) were incubated in six-well plates. Each well was added $2 \mathrm{~mL}$ growth medium consisting of $\alpha$-minimum essential medium (Gibco) supplemented with $16.7 \%$ fetal bovine serum (FBS, Gibco), $1 \%$ glutamine (Sigma), and $1 \%$ penicillin/streptomycin (Sigma). $\mathrm{NPA}_{2}$ coacervates with a final concentration of $5 \mathrm{mg} / \mathrm{mL}$ were added. The control groups were not added $\mathrm{NPA}_{2}$ coacervates. The medium was changed every 2 days. Live/dead staining (Thermo Fisher) was performed on day 7. hMSCs were imaged using a confocal laser scanning microscope (Nikon C2). For the MTT assay, hMSCs $\left(0.6 \times 10^{4}\right.$ cells/ well) were incubated in a 96-well plate in the growth medium. About $5 \mathrm{mg} / \mathrm{mL}$ $\mathrm{NPA}_{2}$ coacervates were used to culture the cells for 7 days. After that, $10 \mu \mathrm{L}$ MTT $(0.5 \mathrm{mg} / \mathrm{mL})$ was added to each well to produce formazan crystals according to the manufacturer's instructions (Thermo Fisher), which were further dissolved by $200 \mu \mathrm{L}$ of DMSO (Sigma). Finally, a Multiskan microplate reader (Thermo Fisher) was used to collect the absorbance value of each well at $540 \mathrm{~nm}$ for cell metabolic calculation.

Animals. Sprague Dawley (SD) rats (200-300 g, female, 8-12 weeks) were obtained from the Laboratory Animal Services Centre (Chinese University of Hong Kong) and cohoused for a week before being randomly assigned to different groups. Animal experiments were approved by the Animal Experimentation Ethics Committee of the Chinese University of Hong Kong (19-152-MIS) and were carried out in accordance with the guidelines of the Animals Ordinance (Chapter 340), Department of Health, Hong Kong.

Oral toxicity test. SD rats (200-300 g, female, 8-12 weeks) receiving oral administration of $\mathrm{NPA}_{2}$ coacervate $(4 \mathrm{~g}$ per $\mathrm{kg}$ rat) on days $1,3,5,7$, and 9 during the 10-day toxicity evaluation and untreated healthy rats were used as the control group. Body weights were measured. Any abnormal symptoms such as diarrhea were recorded.

Ex vivo adhesion experiments. Fresh intestinal tissues were bought from Sha Tin Market (Hong Kong), stored on ice, and washed with PBS buffer $(1 \times)$ three times before use. For ex vivo adhesion testing, $\mathrm{NPA}_{2}$ coacervate stained by Fast Green FCF (Sigma) was deposited on the upright wet intestinal mucosa surface to monitor gravity-induced fluid adhesion behaviors of $\mathrm{NPA}_{2}$ coacervate over time Then the above $\mathrm{NPA}_{2}$ coacervate-coated intestinal tissues were soaked in simulated gastric fluid $(\mathrm{Ga})$ or simulated intestinal fluid (In) at $37^{\circ} \mathrm{C}$ for $2 \mathrm{~h}$ respectively. To monitor changes of the adherent coacervate coatings, photos were taken before and after the soaking and analyzed by Image) 1.8.0_172. Simulated gastric fluid (Ga) and simulated intestinal fluid (In) with enzymes were prepared following United States Pharmacopoeias (USP).

Digestive enzyme-resistant test. $\mathrm{NPA}_{2}$ coacervates were incubated in simulated gastric fluid ( $\mathrm{Ga}$ ) and simulated intestinal fluid (In) at $37^{\circ} \mathrm{C}$ for 3 days, respectively. GPC (Agilent system, 1260 Infinity II) with a refractive index detector was used to detect whether the degraded or dissolved $\mathrm{NPA}_{2}$ coacervate components existed in the simulated body fluids $\left(\mathrm{Ga}^{\prime}, \mathrm{In}^{\prime}\right)$ after 3 days of incubation. PBS $\left(1 \times\right.$, Gibco) was used as the elution phase. $\mathrm{NPA}_{2}$ coacervate was also placed in 1x PBS buffer with pig bile salt $(25 \mathrm{mg} / \mathrm{ml}$, Solarbio) for $24 \mathrm{~h}$ at $37^{\circ} \mathrm{C}$ to test its stability.

In vivo NPA coacervates adhesion experiments. Sprague Dawley (SD) rats (female, 8-12 weeks) weighing around $250 \mathrm{~g}$ were housed in groups of three rats per cage. SD rats were allowed unrestricted access to water and a standard laboratory diet before and after oral gavage. SD rats weighing around $250 \mathrm{~g}$ received 4.5\% Dextran sodium sulfate (DSS, $40 \mathrm{kDa}$, Alfa Aesar) supplemented in the drinking water for 7 days to induce colitis, followed by normal water during the treatment. Healthy SD rats or DSS-induced colitic rats received a single oral gavage of $1.0 \mathrm{ml} \mathrm{NPA}{ }_{2}$ or $\mathrm{NPA}_{3}$ coacervate (modified with a Cy7 tag, a near-infrared 
fluorescent dye). Briefly, an amine-NCO reaction between Cyanine 7 amine ( $0.2 \mathrm{mg}$, Lumiprobe) and polymerized PEG with terminated NCO groups (1 at Supplementary Method 1) was used to modify the hydrophilic PEG shell of NP nanoparticles with a Cy7 tag. Then SD rats were sacrificed after $0,6,24$, or $48 \mathrm{~h}$, and GI tracts were harvested to evaluate the fluorescence retention by an in vivo live imaging system IVIS200 (Xenogen) with an ICG filter channel to measure the fluorescent signal intensity.

In vitro and in vivo drug release. For in vitro Dex-P release, $2 \mathrm{ml}$ of Dex-P in PBS $(1.5 \mathrm{mg} / \mathrm{ml}, \mathrm{TCI})$ was added to $1 \mathrm{ml} \mathrm{NPA} \mathrm{N}_{2}$ coacervate followed by vortex for $15 \mathrm{~s}$ for Dex-P encapsulation. The changes of supernatant Dex-P concentrations were measured by a Shimadzu UV-3600 UV-vis-NIR spectrophotometer at $242 \mathrm{~nm}$. Then $2 \mathrm{ml}$ fresh PBS buffer $(1 \times$, Gibco) was added to the as-prepared Dex-P-laden $\mathrm{NPA}_{2}$ coacervate $(1 \mathrm{ml})$. Dex-P concentrations in the above PBS were measured at $242 \mathrm{~nm}$. The protocols for in vitro release of other first-line small molecule drugs used to treat IBD including antibiotic metronidazole (Metro, J\&K), antiinflammatory 5-aminosalicylic acid (5-ASA, J\&K), and immunoregulatory methotrexate disodium salt (MTX, Alfa Aesar) were the same as in vitro Dex-P release, but the release of Metro, 5-ASA, and MTX was measured at 320, 331, and $371 \mathrm{~nm}$, respectively.

For in vivo Dex-P release, healthy SD rats or DSS-induced colitic rats (200-300 g, female, 8-12 week) received a single oral gavage of $1.0 \mathrm{ml}$ as-prepared Dex-P-laden $\mathrm{NPA}_{2}$ coacervate (Dex-P/NPA ${ }_{2}$ ) or Dex-P aqueous solution (Dex-P/ PBS). The total dosage of Dex-P administered in the two groups was the same $(\sim 1.15 \mathrm{mg})$. SD rats were allowed unrestricted access to water and a standard laboratory diet before and after oral gavage. Blood was collected from the tail vein at selected time points after oral gavage of Dex-P-laden $\mathrm{NPA}_{2}$ coacervate or Dex-P aqueous solution to measure the serum Dex concentrations by a commercial Dexamethasone ELISA assay (Neogen Corporation) following the manufacturer's instructions.

DSS-induced rat models of colitis. SD rats (female, 8-12 weeks) weighing around $250 \mathrm{~g}$ received $4.5 \%$ Dextran sodium sulfate (DSS, $40 \mathrm{kDa}$, Alfa Aesar) supplemented in the drinking water for 7 days to induce colitis, followed by normal water during the treatment ${ }^{25}$. Healthy SD rats were provided with normal water only. Then $\mathrm{NPA}_{2}$ coacervate only, Dex-P-laden $\mathrm{NPA}_{2}$ coacervates $($ Dex-P/NPA 2$)$ or the equivalent amount of Dex-P (1.15 mg, MCE, USA) in PBS (Dex-P/PBS) on days 1,3 , and 5 was administered via an oral route into colitic $\mathrm{SD}$ rats. We have also tried to treat DSS-induced colitic rats with the equivalent amount of Dex-P (1.15 mg, MCE, USA) in PBS (Dex-P/PBS) via enema on days 1,3 , and 5. Dex-P was used at $1.15 \mathrm{mg}$ per dose based on the published reports related to treatments in rodent colitis models ${ }^{52,70,71}$. Untreated colitic SD rats were used as a negative control. All SD rats were allowed unrestricted access to water and a standard laboratory diet before and after oral gavage. All SD rats were sacrificed on day 7 , and the entire colon was excised and gently washed with physiological saline for further evaluation of colon weight and length, histological severity, IBD-associated colonic myeloperoxidase (MPO)-activity, expression of related mRNAs, immunofluorescence staining against tight junction-associated proteins including ZO-1 and occludin-1 (Santa Cruz), and ELISA tests for IL-6 and TNF (MultiSciences).

On day 5 of the treatment, colon tissues from colitic rats receiving Dex-P/NPA or Dex-P/PBS on days 1, and 3 and untreated colitic SD rats (Control) were harvested to study the intestinal immune responses of colitic SD rats by analyzing macrophage polarization. And fresh fecal samples were collected from the above colitic SD rats in each group on day 5 and stored at $-80^{\circ} \mathrm{C}$ in autoclaved sterile vials for further analysis of gut microbiota.

Immunol staining. Immunohistochemical staining for macrophage polarization was performed using an antibody against CD206 (M2 marker, Abcam, ab64693, dilution of 1:200) or iNOS (M1 marker, Abcam, ab283655, dilution of 1:200), followed by Vectastain ABC Kit (Vector Laboratories) and the DAB (3,3-diaminobenzidine) Substrate Kit (Vector Laboratories). Briefly, paraffin sections (7- $\mu \mathrm{m}$ thick) of distal colon tissues were deparaffinized and rehydrated. Then antigen retrieval of sections was performed in microwave-assistant hot antigen retrieval buffer $(1 \times$, citrate buffer $\mathrm{pH} 6.0, \mathrm{Abcam})$. Sections were further incubated with $10 \%$ horse serum (Thermo Fisher) for $30 \mathrm{~min}$ to block nonspecific binding sites. Next sections were incubated at $4{ }^{\circ} \mathrm{C}$ overnight with primary antibody directed against CD206 or iNOS (dilution of 1:200), followed by incubation with a biotinylated secondary antibody (Abcam, ab207995, dilution of 1:500). The staining was further developed using the Vectastain $\mathrm{ABC}$ Kit (Vector Laboratories) and the DAB (3, 3-diaminobenzidine) Substrate Kit (Vector Laboratories) for peroxidase. Finally, sections were stained with hematoxylin.

Immunofluorescence staining for tight junction-associated proteins was performed using antibodies against ZO-1 (Santa Cruz, sc-33725) and occludin-1 (Santa Cruz, sc-271842), respectively. After rehydrating and performing antigen retrieval of the paraffin sections $(7-\mu \mathrm{m}$ thick) of distal colon tissues, nonspecific binding sites were blocked by $10 \%$ horse serum (Thermo Fisher) for $30 \mathrm{~min}$. Next, sections were incubated at $4{ }^{\circ} \mathrm{C}$ overnight with primary antibodies directed against ZO-1 conjugated with Alexa Fluor 488 (dilution of 1:200) and occludin-1 conjugated with Alexa Fluor 594 (dilution of 1:200). Nuclei were then stained with DAPI (4',6-diamidino-2-phenylindole) (Thermo Fisher, dilution of 1:1000). Immunofluorescence images were obtained by using a confocal laser scanning microscope (Nikon C2).

Statistical analysis. All data were presented as mean \pm standard deviation. Statistical analyses were performed by using ordinary one-way ANOVA with Tukey's post hoc testing or two-tailed Student's $t$-test to compare multiple groups or two groups (Graphpad Prism 7.0), respectively. $P$ values less than 0.05 were considered as statistically significant differences among the compared groups, to which different asterisks were assigned $\left({ }^{*} p<0.05 ;{ }^{*} p<0.01\right.$; $\left.{ }^{* * *} p<0.001\right)$. For all multiple comparisons, compare the mean of each column with the mean of every other column.

Reporting Summary. Further information on research design is available in the Nature Research Reporting Summary linked to this article.

\section{Data availability}

The datasets for gut microbiota generated in this study have been deposited in the GEO repository under accession code "GSE167138”. Ribosomal Database Project (RDP) Classifier is "publicly available [https://sourceforge.net/projects/rdp-classifier/files/rdpclassifier/]". All other relevant data supporting the key findings of this study are available within the article and its Supplementary Information files or from the corresponding author upon reasonable request. Source data are provided with this paper.

Received: 8 February 2021; Accepted: 17 November 2021; Published online: 09 December 2021

\section{References}

1. Ng, S. C. et al. Worldwide incidence and prevalence of inflammatory bowel disease in the 21st century: a systematic review of population-based studies. Lancet 390, 2769-2778 (2017).

2. Torres, J., Burisch, J., Riddle, M., Dubinsky, M. \& Colombel, J.-F. Preclinical disease and preventive strategies in IBD: perspectives, challenges and opportunities. Gut 65, 1061-1069 (2016).

3. Windsor, J. W. \& Kaplan, G. G. Evolving epidemiology of IBD. Curr. Gastroenterol. Rep. 21, 40 (2019).

4. Jairath, V. \& Feagan, B. G. Global burden of inflammatory bowel disease. Lancet Gastroenterol. Hepatol. 5, 2-3 (2020).

5. Vass, P. et al. Drying technology strategies for colon-targeted oral delivery of biopharmaceuticals. J. Control. Release 296, 162-178 (2019).

6. Buchman, A. L. Side effects of corticosteroid therapy. J. Clin. Gastroenterol. 33, 289-294 (2001).

7. Tibbitt, M. W., Dahlman, J. E. \& Langer, R. Emerging frontiers in drug delivery. J. Am. Chem. Soc. 138, 704-717 (2016).

8. Kotla, N. G. et al. Bioresponsive drug delivery systems in intestinal inflammation: State-of-the-art and future perspectives. Adv. Drug Del. Rev. 146, 248-266 (2019).

9. Ahadian, S. et al. Micro and nanoscale technologies in oral drug delivery. Adv Drug Del. Rev. 157, 37-62 (2020).

10. Duraj-Thatte, A. M. et al. Genetically programmable self-regenerating bacterial hydrogels. Adv. Mater. 31, 1901826 (2019).

11. Yuk, H. et al. Dry double-sided tape for adhesion of wet tissues and devices Nature 575, 169-174 (2019)

12. Deng, J. et al. Electrical bioadhesive interface for bioelectronics. Nat. Mater. 20, 229-236 (2020).

13. $\mathrm{Bu}, \mathrm{Y}$. et al. Tetra-PEG based hydrogel sealants for in vivo visceral hemostasis Adv. Mater. 31, 1901580 (2019).

14. Wang, Y. et al. A phenol-amine superglue inspired by insect sclerotization process. Adv. Mater. 32, 2002118 (2020).

15. $\mathrm{Xu}, \mathrm{X}$. et al. Bioadhesive hydrogels demonstrating $\mathrm{pH}$-independent and ultrafast gelation promote gastric ulcer healing in pigs. Sci. Transl. Med. 12 eaba8014 (2020).

16. Liu, S. et al. Enzyme-mediated nitric oxide production in vasoactive erythrocyte membrane-enclosed coacervate protocells. Nat. Chem. 12, 1165-1173 (2020).

17. Jeon, O., Wolfson, D. W. \& Alsberg, E. In-situ formation of growth-factorloaded coacervate microparticle-embedded hydrogels for directing encapsulated stem cell fate. Adv. Mater. 27, 2216-2223 (2015)

18. Tang, T. D. et al. Fatty acid membrane assembly on coacervate microdroplets as a step towards a hybrid protocell model. Nat. Chem. 6, 527 (2014).

19. Sing, C. E. \& Perry, S. L. Recent progress in the science of complex coacervation. Soft Matter 16, 2885-2914 (2020) 
20. Khutoryanskiy, V. V. Supramolecular materials: longer and safer gastric residence. Nat. Mater. 14, 963-964 (2015).

21. Love, C. et al. Reversible $\mathrm{pH}$-responsive coacervate formation in lipid vesicles activates dormant enzymatic reactions. Angew. Chem. 132, 6006-6013 (2020).

22. Wang, Q. \& Schlenoff, J. B. The polyelectrolyte complex/coacervate continuum. Macromolecules 47, 3108-3116 (2014).

23. Chang, L.-W. et al. Sequence and entropy-based control of complex coacervates. Nat. Commun. 8, 1-8 (2017).

24. Lee, Y. et al. Therapeutic luminal coating of the intestine. Nat. Mater. 17, 834 (2018).

25. Martin, J. C., Bériou, G. \& Josien, R. Dextran Sulfate Sodium (DSS)-Induced Acute Colitis in the Rat. In Suppression and Regulation of Immune Responses Ch. 12, pp. 197-203 (Springer, 2016). https://link.springer.com/protocol/ 10.1007\%2F978-1-4939-3139-2 12.

26. Melgar, S. et al. Validation of murine dextran sulfate sodium-induced colitis using four therapeutic agents for human inflammatory bowel disease. Int. Immunopharmacol. 8, 836-844 (2008).

27. Deepankumar, K. et al. Liquid-liquid phase separation of the green mussel adhesive protein Pvfp-5 is regulated by the post-translated dopa amino acid. Adv. Mater. 2103828 (2021). https://onlinelibrary.wiley.com/doi/abs/10.1002/ adma.202103828.

28. Ahn, B. K., Lee, D. W., Israelachvili, J. N. \& Waite, J. H. Surface-initiated selfhealing of polymers in aqueous media. Nat. Mater. 13, 867 (2014).

29. Li, L., Yan, B., Yang, J., Chen, L. \& Zeng, H. Novel mussel-inspired injectable self-healing hydrogel with anti-biofouling property. Adv. Mater. 27, 1294-1299 (2015)

30. Deng, J.-H. et al. $\pi-\pi$ stacking interactions: non-negligible forces for stabilizing porous supramolecular frameworks. Sci. Adv. 6, eaax9976 (2020).

31. Salonen, L. M., Ellermann, M. \& Diederich, F. Aromatic rings in chemical and biological recognition: energetics and structures. Angew. Chem. Int. Ed. 50, 4808-4842 (2011).

32. Li, F. et al. Responsive assembly of upconversion nanoparticles for $\mathrm{pH}-$ activated and near-infrared-triggered photodynamic therapy of deep tumors. Adv. Mater. 30, 1802808 (2018).

33. Liao, H. et al. Dynamic supraparticles for the treatment of age-related diseases. Sci. Bull. 64, 1850-1874 (2019).

34. Zhao, P. et al. Nanoparticle-assembled vacuolated coacervates control macromolecule spatiotemporal distribution to provide a stable segregated cell microenvironment. Adv. Mater. 33, 2007209 (2021).

35. Shin, M. et al. Complete prevention of blood loss with self-sealing haemostatic needles. Nat. Mater. 16, 147 (2017).

36. Liang, S. et al. Paintable and rapidly bondable conductive hydrogels as therapeutic cardiac patches. Adv. Mater. 30, 1704235 (2018).

37. Akram Bhuiyan, M. S. et al. In situ deactivation of catecholcontaining adhesive using electrochemistry. J. Am. Chem. Soc. 142, 4631-4638 (2020).

38. Gan, D. et al. Plant-inspired adhesive and tough hydrogel based on Ag-Lignin nanoparticles-triggered dynamic redox catechol chemistry. Nat. Commun. 10, 1-10 (2019).

39. Grieco, C. et al. Intermolecular hydrogen bonding modulates $\mathrm{O}-\mathrm{H}$ photodissociation in molecular aggregates of a catechol derivative. Photochem. Photobio. 95, 163-175 (2019).

40. Grieco, C., Hanes, A. T., Blancafort, L. \& Kohler, B. Effects of intra-and intermolecular hydrogen bonding on $\mathrm{O}-\mathrm{H}$ bond photodissociation pathways of a catechol derivative. J. Phys. Chem. A 123, 5356-5366 (2019).

41. Zhao, P. et al. Mussel-mimetic hydrogels with defined cross-linkers achieved via controlled catechol dimerization exhibiting tough adhesion for wet biological tissues. Chem. Commun. 53, 12000-12003 (2017).

42. Yang, Y., Wang, X., Yang, F., Shen, H. \& Wu, D. A universal soaking strategy to convert composite hydrogels into extremely tough and rapidly recoverable double-network hydrogels. Adv. Mater. 28, 7178-7184 (2016).

43. He, Q., Huang, Y. \& Wang, S. Hofmeister effect-assisted one step fabrication of ductile and strong gelatin hydrogels. Adv. Funct. Mater. 28, 1705069 (2018).

44. Rose, S. et al. Nanoparticle solutions as adhesives for gels and biological tissues. Nature 505, 382 (2014).

45. Zhao, Y. et al. Bio-inspired reversible underwater adhesive. Nat. Commun. 8, 2218 (2017).

46. Liu, J., Tan, C. S. Y. \& Scherman, O. A. Dynamic interfacial adhesion through cucurbit [n] uril molecular recognition. Angew. Chem. 130, 8992-8996 (2018).

47. Wang, Y. Y. et al. Addressing the PEG mucoadhesivity paradox to engineer nanoparticles that "slip" through the human mucus barrier. Angew. Chem. 120, 9872-9875 (2008).

48. Kim, K., Kim, K., Ryu, J. H. \& Lee, H. Chitosan-catechol: a polymer with longlasting mucoadhesive properties. Biomaterials 52, 161-170 (2015).

49. Bouten, P. J. et al. The chemistry of tissue adhesive materials. Prog. Polym. Sci. 39, 1375-1405 (2014).

50. Annabi, N. et al. Surgical materials: current challenges and nano-enabled solutions. Nano Today 9, 574-589 (2014).
51. Christensen, F. et al. The use of gamma scintigraphy to follow the gastrointestinal transit of pharmaceutical formulations. J. Pharm. Pharm. 37, 91-95 (1985).

52. Zhang, S. et al. An inflammation-targeting hydrogel for local drug delivery in inflammatory bowel disease. Sci. Transl. Med. 7, 300ra128-300ra128 (2015).

53. Hatton, G. B., Madla, C. M., Rabbie, S. C. \& Basit, A. W. All disease begins in the gut: influence of gastrointestinal disorders and surgery on oral drug performance. Int. J. Pharm. 548, 408-422 (2018).

54. Fischer, M., Siva, S., Wo, J. M. \& Fadda, H. M. Assessment of small intestinal transit times in ulcerative colitis and Crohn's disease patients with different disease activity using video capsule endoscopy. AAPS PharmSciTech. 18, 404-409 (2017).

55. Effinger, A., O'Driscoll, C. M., McAllister, M. \& Fotaki, N. Impact of gastrointestinal disease states on oral drug absorption-implications for formulation design-a PEARRL review. J. Pharm. Pharm. 71, 674-698 (2019).

56. Fedorak, R. N. et al. Colonic delivery of dexamethasone from a prodrug accelerates healing of colitis in rats without adrenal suppression. Gastroenterology 108, 1688-1699 (1995).

57. Ahn, B. K. et al. High-performance mussel-inspired adhesives of reduced complexity. Nat. Commun. 6, 8663 (2015)

58. Seo, S. et al. Microphase behavior and enhanced wet-cohesion of synthetic copolyampholytes inspired by a mussel foot protein. J. Am. Chem. Soc. 137, 9214-9217 (2015)

59. Wilson, D. S. et al. Orally delivered thioketal nanoparticles loaded with TNFa-siRNA target inflammation and inhibit gene expression in the intestines. Nat. Mater. 9, 923-928 (2010).

60. Kang, H. et al. Immunoregulation of macrophages by dynamic ligand presentation via ligand-cation coordination. Nat. Commun. 10, 1696 (2019).

61. Na, Y. R., Stakenborg, M., Seok, S. H. \& Matteoli, G. Macrophages in intestinal inflammation and resolution: a potential therapeutic target in IBD. Nat. Rev. Gastroenterol. Hepatol. 16, 531-543 (2019).

62. Jiang, K. et al. Local release of dexamethasone from macroporous scaffolds accelerates islet transplant engraftment by promotion of anti-inflammatory M2 macrophages. Biomaterials 114, 71-81 (2017).

63. Kumar, M., Gupta, P., Bhattacharjee, S., Nandi, S. K. \& Mandal, B. B. Immunomodulatory injectable silk hydrogels maintaining functional islets and promoting anti-inflammatory M2 macrophage polarization. Biomaterials 187, 1-17 (2018).

64. Sang, X. et al. Macrophage-targeted lung delivery of dexamethasone improves pulmonary fibrosis therapy via regulating the immune microenvironment. Front. Immunol. 12, 393 (2021).

65. Aras, S. \& Zaidi, M. R. TAMeless traitors: macrophages in cancer progression and metastasis. Br. J. Cancer 117, 1583-1591 (2017).

66. Lee, Y. et al. Hyaluronic acid-bilirubin nanomedicine for targeted modulation of dysregulated intestinal barrier, microbiome and immune responses in colitis. Nat. Mater. 19, 118-126 (2019).

67. Schluter, J. et al. The gut microbiota is associated with immune cell dynamics in humans. Nature 588, 303-307 (2020).

68. Ni, J., Wu, G. D., Albenberg, L. \& Tomov, V. T. Gut microbiota and IBD: causation or correlation? Nat. Rev. Gastroenterol. Hepatol. 14, 573 (2017).

69. Nishida, A. et al. Gut microbiota in the pathogenesis of inflammatory bowel disease. Clin. J. Gastroenterol. 11, 1-10 (2018).

70. Cannarile, L. et al. Glucocorticoid-induced leucine zipper is protective in Th1mediated models of colitis. Gastroenterology 136, 530-541 (2009).

71. Yuan, H. et al. Anti-inflammatory effect of diammonium glycyrrhizinate in a rat model of ulcerative colitis. World J. Gastroenterol. 12, 4578 (2006).

\section{Acknowledgements}

This work was supported by grants from General Research Fund Grants from the Research Grants Council of Hong Kong (Project no. 14202920 and 14204618; Grant recipient: L.B.). The work was partially supported by Hong Kong Research Grants Council Theme-based Research Scheme (Ref. T13-402/17-N; Grant recipient: L.B.)

\section{Author contributions}

P.Z., X.F.X., X.X., P.W.Y.C., and L.B. designed the study, conducted the analysis, and prepared the manuscript. P.Z. synthesized coacervate and performed coacervate characterization procedures. X.F.X., K.K.C.L., and A.R. performed the in vivo experiments P.Z., X.X., X.F.X., and B.Y. performed the handling of colon tissues and fecal samples in vitro. Y.D., P.S., H.Z., H.L., and X.P. provided discussion and analyzed the experiment. All authors were given the chance to read and edit the paper.

\section{Competing interests}

The authors declare no competing interests. 


\section{Additional information}

Supplementary information The online version contains supplementary material available at https://doi.org/10.1038/s41467-021-27463-6.

Correspondence and requests for materials should be addressed to Philip Wai Yan Chiu or Liming Bian.

Peer review information Nature Communications thanks Venkatakrishna Jala and the other anonymous reviewer(s) for their contribution to the peer review of this work.

Reprints and permission information is available at http://www.nature.com/reprints

Publisher's note Springer Nature remains neutral with regard to jurisdictional claims in published maps and institutional affiliations. (c) (1) Open Access This article is licensed under a Creative Commons Attribution 4.0 International License, which permits use, sharing, adaptation, distribution and reproduction in any medium or format, as long as you give appropriate credit to the original author(s) and the source, provide a link to the Creative Commons license, and indicate if changes were made. The images or other third party material in this article are included in the article's Creative Commons license, unless indicated otherwise in a credit line to the material. If material is not included in the article's Creative Commons license and your intended use is not permitted by statutory regulation or exceeds the permitted use, you will need to obtain permission directly from the copyright holder. To view a copy of this license, visit http://creativecommons.org/ licenses/by/4.0/.

(C) The Author(s) 2021 